\title{
One world versus many: the inadequacy of Everettian accounts of evolution, probability, and scientific confirmation
}

\author{
Adrian Kent因 \\ Centre for Quantum Computation, DAMTP, Centre for Mathematical Sciences, \\ University of Cambridge, Wilberforce Road, Cambridge CB3 OWA, U.K. and \\ Perimeter Institute for Theoretical Physics, 31 Caroline Street North, Waterloo, Ontario, Canada N2L 2 Y5
}

(Dated: July 22, 2013)

\begin{abstract}
There is a compelling intellectual case for exploring whether purely unitary quantum theory defines a sensible and scientifically adequate theory, as Everett originally proposed. Many different and incompatible attempts to define a coherent Everettian quantum theory have been made over the past fifty years. However, no known version of the theory (unadorned by extra ad hoc postulates) can account for the appearance of probabilities and explain why the theory it was meant to replace, Copenhagen quantum theory, appears to be confirmed, or more generally why our evolutionary history appears to be Born-rule typical.

This article reviews some ingenious and interesting recent attempts in this direction by Wallace, Greaves-Myrvold and others, and explains why they don't work. An account of one-world randomness, which appears scientifically satisfactory, and has no many-worlds analogue, is proposed. A fundamental obstacle to confirming many-worlds theories is illustrated by considering some toy many-worlds models. These models show that branch weights can exist without having any role in either rational decision-making or theory confirmation, and also that the latter two roles are logically separate.

Wallace's proposed decision theoretic axioms for rational agents in a multiverse and claimed derivation of the Born rule are examined. It is argued that Wallace's strategy of axiomatizing a mathematically precise decision theory within a fuzzy Everettian quasiclassical ontology is incoherent. Moreover, Wallace's axioms are not constitutive of rationality either in Everettian quantum theory or in theories in which branchings and branch weights are precisely defined. In both cases, there exist coherent rational strategies that violate some of the axioms.
\end{abstract}

\section{INTRODUCTION}

\section{A. Some common ground}

Although I disagree with the Everettian contributors to this volume on some fundamental questions, I think they deserve much credit for developing some creative and interesting ideas and arguments, which have certainly helped advance our understanding of fundamental science. To elaborate on this, let me note some points on which I agree with many Everettians.

First, the Everettian programme had a sensible motivation. Everett asked[1], in effect, whether quantum theory really needs to be framed in such a way that the evolution of the wave function is governed by two different laws: generic unitary evolution together with the projection postulate when measurement takes place. It's a good question. Even if, rather than the projection postulate, quantum theory came equipped with a precise extra dynamical law implying the postulate as an approximation, it would be natural to ask if we really needed it. As it is, there is quite a compelling case for exploring whether we can make sense of purely unitary quantum theory.

Second, it is a sensible project to try to extract a physical ontology from a unitarily evolving quantum state vector, given a theory of the initial state or initial conditions, a Hilbert space defining a representation of position, momentum and other canonical operators, and a dynamical theory that expresses the Hamiltonian in terms of these operators. Whether the project succeeds in producing an ontology with the properties that Everettians fondly imagine is another question - but certainly one worth discussing. One still, strangely, sometimes hears the argument that it is illegitimate - a basic misunderstanding of quantum theory - even to examine the possibility of giving the state vector a direct physical interpretation. This seems to me simply unimaginative dogma. Everettians are right to insist that their programme should be judged on whether or not it works, not on whether it respects pre-Everettian quantum orthodoxies.

*a.p.a.kent@damtp.cam.ac.uk 
Third, neither the apparently fantastic nature of the Everettian worldview, nor the superficial conflict between postulating multiple independent mutually inaccessible worlds and Occam's razor, are entirely compelling arguments against the Everett programme. One needs to consider Everettian ideas in the context of other attempts to make sense of quantum theory, and in detail. One of the great intellectual challenges of theoretical physics is to find a mathematically elegant, universally applicable, Lorentz covariant, scientifically adequate version of quantum theory that supplies a well-defined realist ontology. If the Everett programme really could produce a well-defined Lorentz covariant physical ontology that adds little or no arbitrary structure to the mathematics of quantum theory, and that reproduces all the scientific successes of Copenhagen quantum theory within its domain of validity, it would have solved this fundamental problem. Given the present alternatives, we would, I think, at that point, have to consider it seriously as a possible account of reality.

Now, in fact, I think that the Everett programme fails in these ambitions, for reasons explained below. I am also optimistic [3] that we can find simpler one-world versions of quantum theory that have all the aforementioned virtues and none of the problems that afflict, and I think ultimately doom, the Everett programme. But I see no way to make either conclusion so transparently true as to eliminate the need for argument.

Fourth, it matters - it is scientifically important to understand - whether the Everettian programme can possibly succeed. If Everettians really could produce a theory of reality with all the proclaimed virtues, it would clearly weaken (though not eliminate) the motivation for other attempts at solving the quantum reality problem - just as finding a consistent quantum theory of gravity would weaken (though not eliminate) the motivation for looking for others. Conversely, if, as I argue, the Everettian programme has fairly definitely failed, then the problem of finding a viable formulation of quantum theory applicable to closed quantum systems looms rather large among the concerns of theoretical physics. The failure of the Everett programme adds to the likelihood that the fundamental problem is not our inability to interpret quantum theory correctly but rather a limitation of quantum theory itself. If so, my guess is that we most likely won't find an adequate cosmological theory so long as we assume that quantum theory is universally valid - so we should be looking for possible signals of the failure of quantum theory applied to the universe. Likewise, if so, quantum interference quite likely breaks down somewhere between the microscopic and the macroscopic - so we should be working harder to characterize the most promising types of experiment to test this.

Everettian ideas have been around for fifty years, and influential for at least the last thirty. Yet there has never been a consensus among theoretical physicists either that an Everettian account of quantum theory can be made precise and made to work, or that the Everettian programme has been comprehensively refuted. These questions are quite central to the future of theoretical, experimental and observational physics. We need to resolve them and move forward.

\section{B. Everett's elusive essence}

"When he died, his heirs found nothing save chaotic manuscripts. His family, as you may be aware, wished to condemn them to the fire; but his executor - a Taoist or Buddhist monk - insisted on their publication."

"We descendants of Ts'ui Pên," I replied, "continue to curse that monk. Their publication was senseless. The book is an indeterminate heap of contradictory drafts."

(Jorge Luis Borges, The Garden of Forking Paths [4])

"... so crowded with ... empty sophistication that it is extremely difficult to perceive the simple errors at the basis. It is like fighting the hydra - cut off one ugly head, and eight formalizations take its place."

(P.K. Feyerabend, How to Defend Society Against Science [5])

After fifty years, there is no well-defined, generally agreed set of assumptions and postulates that together constitute "the Everett interpretation of quantum theory". Far from it: Everett[1, 2], DeWitt[7], Graham [8], Hartle [6], Geroch 10], Deutsch [11], Deutsch 12], Saunders 13], Barbour 14] (partly inspired by Bell[15], though Bell's aim was not to inspire), Albert-Loewer 16], Coleman [17], Lockwood 18], Wallace 19], Wallace[20], Vaidman [21], Papineau [22], Greaves [23], Greaves-Myrvold [24], Gell-Mann and Hartle [25], Zurek 26] and Tegmark 27], among many others, have offered distinctive and often fundamentally conflicting views on what precisely one needs to assume in order to get the Everett programme off the ground, and what precisely an Everettian (or, some say, post-Everettian) version of quantum theory entails.

I am primarily interested here in contrasting realist "one-world" and "many-worlds" accounts of quantum theory. By one-worlders, I mean those who aim to find a version of quantum theory in which quantum experiments really have only one outcome, we really have only one version of our future selves at any future time, and some intrinsic randomness in nature determines which outcome occurs and which future self is realised from among the range of possibilities defined by the theory. For example, within its domain of validity, the Copenhagen interpretation of 
quantum theory is a one-world theory. By many-worlders, I mean those who share Everett's view that a unitarily evolving quantum state vector should be interpreted as directly representing reality, and the future versions of ourselves that observe different outcomes of quantum experiments should be interpreted as equally real.

So, I will not discuss here attempts at "post-Everettian" interpretations like those of Gell-Mann and Hartle 25] and Zurek [26], which fall into neither camp, and seem - despite much critical probing - unclear on, or uncommitted to taking a stance on, precisely what, if anything, in the theory corresponds to objective external reality. (Extensive critiques of Gell-Mann and Hartle's approach can however be found elsewhere 28 33].)

My main focus is on the recent attempts by Wallace [19, 20], Greaves and Myrvold[24], and, to a lesser extent, Papineau [22] and Saunders [13], to define, analyse and test realist many-worlds interpretations. These authors offer different, and on some points mutually inconsistent, approaches, but nonetheless share enough common perspectives to be considered together. Their papers include some very interesting and creative arguments, which raise important scientific questions. However, I will argue below that none of their approaches produces a scientifically adequate version of quantum theory.

Shadowing these discussions is the spectre of the 'many-minds interpretation' set out some time ago by Albert and Loewer [16]. Essentially everyone, including Albert and Loewer, agrees that the many-minds interpretation, while logically consistent and in accord with the data, is utterly unsatisfactory, since it adds to the Everettian formalism a collection of ad hoc postulates which not only are (even by Everettian standards) fantastic, but also undercut the motivation for taking Everett seriously, namely that it purports to explain how to make sense of quantum theory without adding extra equations or interpretational postulates. So, no one - certainly no one represented in this volume - wants to be a many-minder. And here lies the problem: it seems to me (and to others - see in particular Albert's contribution 34] to this volume) that, at various points in their arguments, Saunders, Wallace, Greaves-Myrvold and Papineau tacitly - and, since they reject the many-minds interpretation, illegitimately - appeal to many-minds intuitions. Indeed, at least in the first three cases, it seems to me that if one fleshed their ideas out into a fully coherent and complete interpretation, one would necessarily arrive either at the many-minds interpretation or something even worse. I will elaborate on this below.

Of course, these discussions crucially turn on our understanding of what counts as scientifically adequate. The idea that reality contains many essentially independent quasiclassical worlds corresponding to different possible cosmological and experimental outcomes clearly isn't, per se, susceptible to logical refutation. That isn't at issue. The key question, to my mind - and I think modern Everettians, including the authors considered here, generally agree - is whether we can find an appealingly simple version of quantum theory in which a realist many-worlds ontology is essential (i.e. there is no equally simple one-world variant) and which (at minimum) replicates all the scientific successes of one-world quantum theory (i.e. quantum theory including some form of the projection postulate, or some principle from which it can, approximately and within a suitable domain of validity, be derived). I believe that we can't. In particular, it seems to me the Everettian programme has not produced and cannot produce a scientifically adequate alternative account that reproduces the standard one-world account of probabilistic inferences derived from quantum theory - despite the ingenious recent attempts of contributors to this volume.

Some commentators sympathetic to the Everettian programme 22, 24] argue that a double standard is at work here: that criticisms of the Everettian programme's attempt to account for the appearance of probability can and should equally well be applied to the standard understanding of the role of probability in one-world versions of quantum theory, and indeed of probabilistic scientific statements in general. To respond to this point, I consider below some fundamental differences between randomness (or apparent randomness) in one-world quantum theory and its purported Everettian analogue, and point out what seem to me irresolvable problems with the latter.

\section{ONE-WORLD THEORIES AND PROBABILITY}

Copenhagen quantum theory is a one-world version of quantum theory: any given experiment or quantum event has a number of possible outcomes, but only one actual outcome. Some other non-Everettian variants and modifications of quantum theory, such as de Broglie-Bohm theory and dynamical collapse models, similarly randomly select from many possible physical evolutions, and can be (and usually are) interpreted as defining a unique quasiclassical world. The consistent histories approach [25], if combined with an (alas unknown) suitable set selection rule, would also lead naturally to a one-world interpretation, in which reality is described by one randomly chosen history from the selected set. And these by now venerable contenders certainly don't exhaust the possible options. [3] My aim here is not to advocate a specific one-world version or variant of quantum theory, or to assess the current state of the art, but rather to compare and contrast one-world and many-world accounts of probability. For that purpose, let us suppose, for the sake of argument, that we have to hand a particular one-world theory that implies that, while the universe

could have evolved in a (presumably very large) number of different ways, one quasiclassically evolving world - the one we observe - was randomly selected. 
One-world versions of quantum theory, together with hypotheses about the initial conditions and unitary evolution, predict the probabilities of our experimental results and observations. We test the theory and these hypotheses by checking whether the results are of a form we would typically expect given the predicted probabilities. In practice, pretty much everyone agrees on the methodology of theory confirmation, at least sufficiently so that, for example, everyone agrees that, within the domain of validity of Copenhagen quantum theory, the Born rule is very well confirmed statistically. However, there is much less agreement on how, or even whether, we can make sense of fundamentally probabilistic physical theories. What exactly, if anything, does it mean to say that the probability of the universe turning out the way it did was 0.00038 ?

Everettian authors have stressed this last point lately. We should not, they argue, apply different standards to one-world and many-worlds quantum theory. If our account of standard probability applied to one-world quantum theory is suspect, or incomplete, or involves ad hoc postulates, we cannot reasonably reject an alternative many-worlds account on the grounds that it runs into difficulties that might, on close analysis, turn out to be precisely analogous.

There are several possible responses for one-worlders here. One response is to try to defend or buttress or further develop frequentism, or another standard account of standard probability. A second is to try to point out some insuperable problems with many-worlds accounts of probability, and thus make the case that, whatever difficulties one-world quantum theory might run into, many-worlds quantum theory cannot possibly be satisfactory. A third is to argue that the difficulties that many-worlders face in dealing with probability are worse than - not, as claimed, precisely analogous to - those faced by one-worlders.

I think the first of these options is worth pursuing. I think too that the second and third lines of argument are valid, and I will develop them later. But, in this section, I want to make a separate point. I want to suggest a nonstandard account in which the scientific space usually occupied by one-world probabilistic theories is filled instead by deterministic theories with a large amount of theoretically unspecified data. This allows us to compare, verify and falsify theories, and to recover essentially all of current science, without assigning a fundamental role to probability per se. Convinced believers in a chancy world might regard this as a useful fall-back position pending a fully satisfactory explanation of standard probability. It might, alternatively, be seen as an account with enough attractions of its own that it could be preferable to any standard account involving probability. Either way, it offers a way of making scientific sense of one-world quantum theory that has no many-worlds analogue.

Consider a probabilistic theory $T$, and suppose for simplicity that it predicts a finite set of probabilistic events, labelled by the index $i$, each with finitely many possible outcomes $x_{i}^{j}$, labelled by the index $j \in J_{i}$, for which it predicts nonzero probabilities $p_{i}^{j}$. For simplicity, we also suppose for the moment that the possible outcomes for any given event, and their probabilities, are independent of the outcome of any other event. We say two events $i$ and $i^{\prime}$ are of the same type, according to $T$, if the sets $\left\{p_{i}^{j}\right\}$ and $\left\{p_{i^{\prime}}^{j}\right\}$ are identical. Let $B=\{0,1\}, B^{*}=\{\emptyset, 0,1,00,01, \ldots\}$ be the set of finite binary strings, and $B^{r}$ the set of length $r$ binary strings. Let $n=\prod_{i}\left|J_{i}\right|$ be the size of the list of possible sets of outcomes, which we write as $N=\{1,2, \ldots, n\}$.

A length $r$ code for the outcomes is any surjective map $C: B^{r} \rightarrow N$. Given such a code, we can define an alternative probabilistic theory $T^{C}$ by stipulating that a binary string $b$ in $B^{r}$ is randomly chosen from the uniform distribution, and that the outcomes are given by $C(b)$. By taking $r$ sufficiently large, and choosing $C$ so that $|\{b: C(b)=i\}| \approx 2^{r} p(i)$ for each $i \in N$, we can find theories $T^{C}$ whose probability assignments are arbitrarily close to those of $T$.

A length $r$ subcode for the outcomes is any map (not necessarily surjective) $C: B^{r} \rightarrow N$. Again, given a subcode, we can define a probabilistic theory $T^{C}$ as above: here $T^{C}$ may assign zero probability to some outcomes for which $T$ assigns non-zero probability.

We can define another type of theory from the triple $\left(T^{C}, C, r\right)$ : a theory that simply states that the data will be those predicted by $T^{C}$ and $C$ given some length $r$ binary string as input, and makes no prediction about the binary string. We call this theory $D\left(T^{C}, C, r\right)$, using $D$ to emphasize that we now regard the theory as deterministic. One might view the binary string in $D\left(T^{C}, C, r\right)$ as playing a role analogous to a constant of nature in a deterministic theory: its value is not fixed by the theory, and can only be determined empirically. In this case, even if the map $C$ is injective, determining the entire string would require observing every random event in the universe.

Now, on the view that there is a unique "correct" fundamentally probabilistic theory of nature $T$, each probabilistic theory of the form $T^{C}$ must be either equivalent to $T$ (which is possible only if the probabilities $p_{i}^{j}$ are all dyadic), or else incorrect (though possibly a good approximation to $T$ ). Note though that, given a finite set of data, many other probabilistic theories besides $T$, including some of the form $T^{C}$, will be consistent with the data. Indeed, we would generally expect some theories $T^{\prime}$ to fit the data better than $T$, in the sense that the same sets of events are of the same type according to $T$ and $T^{\prime}$, and the probabilities $p_{i}^{\prime j}$ are closer than $p_{i}^{j}$ to the observed relative frequencies for events of the same type. If we nonetheless regard $T$ as likelier to be correct than $T^{\prime}$, it must be for reasons other than purely empirical - presumably on grounds of elegance or simplicity. And if we maintain that there is a unique correct fundamental theory, it seems to follow that the correct theory is determined by a set of probabilities $\left\{p_{i}^{j}\right\}$ not determined by the physical universe (although perhaps very well approximated by relative frequencies of physical events). 
Here's an alternative view. It may be, if not meaningless, then at least unnecessary, to appeal to the idea of a unique correct fundamentally probabilistic theory of nature, or even to define probability as a fundamental physical concept. Instead of considering probabilistic theories $T^{C}$, we can compare deterministic theories $D\left(T^{C}, C, r\right)$ against one another and against the data. In evaluating these theories, we use the criteria of simplicity and elegance. These criteria have no precise mathematical definition. They include judgements about the form of $T^{C}$ and $C$, as well as the parameter $r$ (which is a precise measure of complexity for the part of the theory defined by the unknown binary string). In saying that one theory $D\left(T^{C}, C, r\right)$ is our best current theory - or perhaps that our best descriptions of nature are given by a class of similar such theories - we mean that we can't presently find a substantially simpler and more elegant theory that fits the data. The stronger meta-theoretic hypothesis that a theory $D\left(T^{C}, C, r\right)$ is, up to approximate equivalence, the best theory of nature implies that, given all the physical data in the universe, one would not be able to find a simpler, more elegant, compelling theory.

This could be made more quantitative by formalising the discussion within the context of a fixed model of computation, for instance a (classical) Turing machine. (This is why we have chosen to consider theories with unknown binary strings, although of course bases other than binary could also be used.) Here, a theory is a program for generating a mathematical representation of the complete set of physical data. A theory with unknown data is a program that requires an unknown input string of stipulated length. The theory's simplicity depends, inter alia, on both the length of the program and the length of the required input string. Each of these is a natural simplicity parameter. The halting time of the program is another significant parameter, which gives one way of quantifying the elegance of a theory.

In principle, within a fixed computation model, it's possible to carry out an exhaustive search of all theories with total length $\leq L$ that halt after $\leq N$ steps. In principle, thus, given all the physical data, one can test the hypothesis that $D\left(T^{C}, C, r\right)$ is the best theory among all those whose program and input strings satisfy given length bounds, and which satisfy other stipulated simplicity and elegance constraints, that halt after any given finite time, relative to a fixed computation model.

Thus, instead of talking about a probabilistic physical theory that produces a random set of physical data, we can consider a deterministic physical theory whose definition includes a set of pre-determined but a priori unknown physical data, together with the meta-theoretic hypothesis that this description is essentially algorithmically incompressible. If we learn empirically that the data are in fact significantly compressible, then this hypothesis is refuted, and we may replace the theory by a more economical one.

It should be stressed that these measures of simplicity and elegance are by no means intended to be an exhaustive list. For example, another elegance criterion is given by the principle of scientific induction, which suggests we should prefer a theory that suggests that a hitherto apparently fair coin will continue to be apparently fair over one that suggests that it will henceforth always come up heads, even though the latter theory requires a shorter input string (and so is simpler by one of the above measures). 53. Comparing scientific theories generally involves a wide and arguable variety of quantitative and qualitative simplicity and elegance criteria, and nothing in this account alters that: the aim here is only to propose a different treatment of apparent randomness when comparing theories.

\section{A. Example: reinterpreting a fair coin}

For example, in a universe with an apparently random process that apparently mimics a fair coin and produces a large number $N$ of apparently independent outcomes, our meta-theoretic hypothesis might suggest that we cannot find a simpler correct theory than one that states that the length $N$ binary string is essentially algorithmically incompressible. If, in fact, the string $S$ turns out to consist of $0.01 N$ zeroes and $0.99 N$ ones, we can certainly generate a more economical theory, and this hypothesis is refuted.

According to the standard account of probabilistic theories, if a probabilistic theory $P T$ says that zeroes and ones are equiprobable and independently generated, the outcome $S$ is extremely improbable, but not logically impossible. The theory $P T$ is thus not logically refuted by the outcome $S$. In practice we would reject it - but, without a fundamentally satisfactory account of probability, it is hard to give a completely satisfactory justification for doing so.

In our alternative account, however, no such problem arises. Our hypothesis predicts that a given physical dataset is essentially incompressible - where "essentially" incorporates some judgements about the tradeoffs between small gains in compression of the dataset and simplicity and elegance in other aspects of the theory. If the dataset turns out to be a string such as $S$ that is significantly compressible, so that we can fit the data by a simpler theory, the hypothesis is falsified and the original theory replaced. 


\section{B. Example: reinterpreting a biased coin}

Now consider a universe with an apparently random process that apparently mimics a coin with bias $p>\frac{1}{2}$ towards zero and produces $N$ apparently independent outcomes. We can then produce theories that state that the length $N$ binary string is compressible to $H(p) N+o(N)$ bits. For example, a theory which says that the length $N$ string will contain between $p N-10 \sqrt{N}$ and $p N+10 \sqrt{N}$ zeroes has the required compression, since we can binary code all such strings in a code of length $H(p) N+o(N)$. Clearly there are many somewhat similar such theories - the string contains between $p N-9 \sqrt{N}$ and $p N+9 \sqrt{N}$ zeroes, between $p N-11 \sqrt{N}$ and $p N+11 \sqrt{N}$ zeroes, and so on. On this view of scientific accounts of apparently random data, that's the best one can hope for: generically, no single clearly optimal theory will emerge. However, we can hypothesize that theories of roughly this length are essentially best possible - i.e. that the string cannot be compressed to significantly shorter than $H(p) N$ bits - and this hypothesis is testable and falsifiable.

Again, these theories reproduce deterministically predictions that the standard probabilistic theory says hold with probability very close (but not equal) to one. They exclude some very low probability events which would, if realised, in practice persuade almost everyone that the probabilistic theory was wrong, even though their occurrence is logically consistent with the theory.

\section{Conclusion}

According to this account, we should consider one-world quantum theory as a theory which requires a binary string as input, and consider it alongside the meta-theoretic hypotheses that (a) there is no significantly more compressed description of the data obtained from quantum experiments than that given by encoding them in binary, using a coding that would produce an approximately uniform distribution over binary strings if the data were probabilistically generated via the Born rule, (b) the data can indeed be thus described. If one of these hypotheses turns out to be incorrect - if, for example, the data in all Bell experiments consistently show significantly greater violations of the CHSH inequality than quantum theory predicts - then we must find a better theory. Conversely, the theory logically (not merely with high probability) implies that we will see no consistent regularities in our experimental data that would, on the usual account, be highly improbable.

Among the scientific virtues of this account, as I see it, are its explicitness about the provisional nature of our theories, and its undogmatic sidestepping of the problem of giving a fundamental meaning to probability. It recognizes the possibility that random-seeming data may turn out to have a simpler description. It recognizes too that, if we find consistent regularities that a probabilistic theory says are highly improbable, then we should and will feel impelled to produce a better theory. At the same time, it stays silent on the question of whether random-seeming physical data are genuinely randomly generated in some fundamental sense, and hence avoids the need to explain what such an assertion could really mean and how we could be persuaded of its truth.

One-world quantum theory, read in this way, allows us to draw logical inferences about the physical world. It predicts - it is not merely consistent with the fact - that there will be no regularities in the data of a type that would allow for a significantly simpler theoretical description. If that prediction turns out to be wrong, the theory is refuted. Interpreted thus, one-world quantum theory can be read as a well-formulated scientific theory, in a way that allows a straightforward account of scientific confirmation and refutation. If we assume it is correct, we have an explanation for the apparent fact that our evolutionary and experimental histories contain no regularities that would be inexplicably improbable according to the Born rule. To the extent that the project outlined above can be fleshed out and succeeds - and I am optimistic that it can and does - proponents of one-world quantum theory can rest relatively easy on the question of randomness.

\section{TOY MANY-WORLDS THEORIES AND THEIR USES}

If we knew of probability theory only through its use in Copenhagen quantum theory - if we had no familiarity with coin tosses, dice rolls, noise, or any other effectively unpredictable classical systems - we would probably be (even more) deeply confused about the nature of both quantum theory and probability. I suspect this is the cause of much of the continuing confusion over many-worlds quantum theory: discussions need simultaneously to grapple with the quite unfamiliar concept of many branching worlds and the specific peculiarities of Everettian quantum theory.

This motivates defining some simpler many-worlds theories. Another reason for doing so is that some key Everettian ideas - for example, Greaves and Myrvold's attempt [24] at an account of many-worlds theory confirmation - can really only sensibly be discussed if we can consider a class of many-worlds theories, not just the single example of Everettian 
quantum theory. Readers may initially find the form of the following theories a little intellectually unsettling, but I recommend persevering: they shed a great deal of light on Everettian arguments.

Let me stress right away that these are not perfect models for Everettian quantum theory. That is, in fact, part of the point: they allow us to separate out general claims about rationality and theory confirmation in multiverse theories from claims that rely on specific features of quantum theory. In particular, they allow us to see why Greaves-Myrvold's account of many-world theory confirmation doesn't work.

\section{A. Some toy multiverses}

The following toy multiverses are all classical, in the sense that the state of any branch at any time is defined by a classical physical theory, and they all have a definite branching structure.

Consider, first, the branching multiverse $C B U_{1}$, which includes conscious inhabitants, and also includes a machine with a red button on it and a tape emerging from it, with a sequence of numbers on it, all in the range 0 to $(N-1)$. Whenever the red button is pressed in some universe within the multiverse, that universe is deleted, and $N$ successor universes are then created. All the successors are in the same classical state as the original (and so, by hypothesis, all include conscious inhabitants with the same memories as those who have just been deleted), except that a new number has been written onto the end of the tape, with the number $i$ being written in the $i$-th successor universe.

Suppose, too, that the multiverse's inhabitants believe that something like this is indeed happening. The numbers on the tape play a significant role in their society. In particular, it is quite common to place bets on future numbers, and social mores ensure that such bets are always honoured. Of course, since one's own universe will be destroyed before the next number is written, placing such a bet means - they correctly believe - redistributing resources amongst one's successors. Some inhabitants may find reasons for preferring some redistributions over others. We need not discuss yet precisely what these reasons and preferences (both of which may be different for different inhabitants) may be.

It might be helpful to imagine that the universes are being run on a simulator by technologically advanced beings, who simply end one simulation whenever the red button is pressed, and then start simulating the successor universes from the appropriate initial states. We will sometimes assume that the inhabitants, indeed, believe this to be the case.

Suppose, further, that some of the inhabitants of $C B U_{1}$ have acquired the theoretical idea that the laws of their multiverse might attach weights to branches, i.e. a number $p_{i}$ is attached to branch $i$, where $p_{i} \geq 0$ and $\sum_{i} p_{i}=1$. They may have different theories about how these weights are defined: for instance, that the weights are always $\left\{p_{i}\right\}$, that they are always $\left\{q_{i}\right\}$, that they vary over time according to some rule, and so on. As it happens, though, these theories are all incorrect: there are no weights attached to the branches. To be clear: this is not to say that the branches have equal weight. Nor are they necessarily physically identical aside from the tape numbers. They may perhaps be distinguished by other features: for example, if they are simulations, they may be simulated by different hardware or software. However, any such differences do not yield any natural quantitative definition of branch weights. There is just no fact of the matter about branch weights in this multiverse.

The multiverse $C B U_{2}$ is similar to $C B U_{1}$. In this universe, there are indeed numbers attached to the branches, but the way they are attached means that they should (by our lights, and also by the inhabitants', if only they understood the full picture) have no significance to any decisions the inhabitants make about bets/redistributions. For instance, we could extend the simulation idea, and imagine that the technologically advanced beings simply choose, on whim, to write the number $p_{i}$ somewhere inconspicuous in the simulation of successor universe $i$, in such a way that it has no effect on the inhabitants, and that it has no other significance.

The multiverse $C B U_{3}$ is similar to $C B U_{2}$. However, this time the numbers attached to the branches by the physical theory are attached in such a way that it can be plausibly argued that they could reasonably play a significant role in the decisions the inhabitants make about bets/redistributions. For instance, we could imagine that when the technologically advanced beings create successor universes, they create not just one successor corresponding to each outcome $i$, but a number of distinct successor universes, all identical apart from their outcome values, and the number containing outcome $i$ is proportional to the weight $p_{i}$. (We assume here the $p_{i}$ are rational numbers.)

\section{B. Some possible strategies}

Consider an inhabitant of any of the above multiverses, who believes that the weight $p_{i}$ is attached to the outcome $i$. Suppose they are offered a variety of bets that give their successor a good $G_{i}$ in a universe in which outcome $i$ obtains, and they (the original inhabitant) attach utility $U_{i}$ to this good. We suppose the $U_{i}$ are finite real numbers, not necessarily positive (the goods may be bads); and, of course, both $G_{i}$ and $U_{i}$ depend on the bet. 
How might they proceed to evaluate and rank such bets? Weight-sensitive inhabitants believe that branch weights exist and should play a role in their betting preferences. Weight-indifferent inhabitants may also believe that the physical theory attaches weights, but if so, do not believe they are of any relevance to a rational betting strategy. (Such an inhabitant might, for example, believe that they live in a multiverse like $C B U_{2}$.) Among their options is to mimic the strategy of a weight-sensitive inhabitant, except that they treat all branch weights as equal. By this means, given any weight-sensitive strategy, we can define a corresponding weight-indifferent strategy. Here are some examples of weight-sensitive strategies:

- The mean utilitarian ranks bets according to the value of $\sum_{i} p_{i} U_{i}$.

- The Price-Rawlsian's dominant concern [35, 36] is with the welfare of their least satisfied future self. They rank bets first according to $\min \left(U_{i}\right)$, and then some list of tie-breaking criteria. To be definite, let's say their next criterion is the value of $\sum p_{j}$, summed over all $j$ such that $U_{j}=\min \left(U_{i}\right)$, followed by $\min \left(U_{j}: U_{j} \neq \min \left(U_{i}\right)\right)$, and so on.

- The future self elitist's dominant concern is that the best possible version of their future self should be realized somewhere; they have little interest in mediocre future selves, whom they regard as losers. Their bet rankings are thus dominated by $\max \left(U_{i}\right)$, and they break ties using the mirror image of the Price-Rawlsian's criteria.

- The rivalrous future self elitist takes things one stage further. Not only do they identify their interests exclusively with those of their best possible future self, but they regard that self as in competition with the others, and feel happiest - all else being equal - if that competition is won by as large a margin as possible. They rank bets first by $\max \left(U_{i}\right)$, then by $\left.\max \left(U_{i}\right)-\max \left(U_{j}: U_{j} \neq \max \left(U_{i}\right)\right)\right)$, and so on.

- The median utilitarian's dominant concern is for median utility. Reordering the index labels so that $U_{1} \leq U_{2} \leq$ $\ldots \leq U_{n}$, let $j$ be such that $\sum_{i=1}^{j-1} p_{i}<\frac{1}{2}$ and $\sum_{i=1}^{j} p_{i} \geq \frac{1}{2}$ : they rank bets first according to the value of $U_{j}$. (They also have some tie-breaking criteria: one option is to break ties by considering the mean utility.)

- The $x$-percentile utilitarian's dominant concern is for the utility of the future self ranked at $x \%$ in the distribution. They proceed like the median utilitarian, with $\frac{1}{2}$ replaced by $\frac{x}{100}$. The Price-Rawlsian, median utilitarian and future self elitist are all special cases.

- The future self democrat believes her preference should be that which would result from a democratic vote among her future selves. Given a finite list of possible bets, for each value of $x$, she asks herself how she would order her preferences among the bets, if she knew that she would become the future self ranked at the $x$-percentile of the elected bet. (The answer might be that her future self's voting preference would always be dominated by its own welfare under this hypothesis, but it need not: it depends whether she cares about the welfare of contemporaneous versions of herself in other branches.) She then tallies the votes, integrating over $x$ using branch weight measure, and using, say, a single transferable vote system. The winner of the vote is her preferred bet. If the election is tied, she has more than one equally preferred bet.

- An example of a future self distribution engineer is someone who seeks to maximise an expression of the form

$$
\sum_{i} f_{1}\left(U_{i}\right) p_{i}+\sum_{i j} f_{2}\left(U_{i}, U_{j}\right) p_{i} p_{j}+\ldots
$$

where the $U_{i}$ are the utilities of future branches with weights $p_{i}$, and $f_{n}$ is some given joint function of $n$ variables. [54]

\section{Many-worlds rationality reconsidered in toy models}

"If you do what you've always done, you'll get what you always got."

(variously attributed)

According to Wallace 20] and Greaves-Myrvold [24], we should define rational behaviour in a multiverse via axioms generalizing those proposed by Savage 41] in order to justify using the standard calculus of probabilities and utility functions for rational decisions in a single world in which future events are uncertain. 


\section{Savagean rationality in one world}

Savage, engagingly and rather admirably, presented his approach to rationality in the presence of (one-world) uncertainty

"... in a tentative spirit, for I realize that the serious blemishes in it apparent to me are not the only ones that will be discovered by critical readers." [42]

Everettian neo-Savageans [20, 24], as I read them, seem rather less self-critical — puzzlingly so, since applying Savagean decision theory to Everettian quantum theory raises many new questions without solving any of the old ones. This raises some general worries, which are developed to some extent elsewhere in this paper, but might also be taken in other directions. First, if Savage's axioms are, in fact, unable to give a completely satisfactory account of ideal rational behaviour in the presence of one-world uncertainty, it seems very unlikely that a completely satisfactory axiomatic treatment of many-worlds rationality can be produced by generalizing them. Second, giving a satisfactory account of ideal rational behaviour in the presence of one-world uncertainty (or some many-worlds generalization) may in any case not be enough. (For one thing, we are not ideal rational agents. For another, as Albert 34] has eloquently stressed, there is a crucial difference between showing that one can find a rational justification for behaving as though the world were a certain way and showing that the world actually is that way.) Third, however far Savage can or cannot guide rational agents in one uncertain world, it isn't obvious that his programme generalizes at all to many-worlds theories in general or to Everettian quantum theory in particular.

\section{Many-worlds rationality according to Greaves-Myrvold}

Let me now focus on Greaves-Myrvold's axioms, which are intended to apply to general many-worlds theories, and so can straightforwardly be considered within the toy models described above. I will consider later Wallace's arguments, which are framed for the special case of Everettian quantum theory, and for the moment simply note that their logic suggests the same conclusions here as Greaves-Myrvold's.

In Greaves-Myrvold's view, the mean utilitarian's strategy is rationally justifiable, and the others are branded irrational, since they violate one or more of the axioms. For example, the future self elitist and the Price-Rawlsian violate their continuity postulate, $P 6$, the median utilitarian violates $P 2$, the future self democrat violates transitivity, $P 1 a$, and the rivalrous future self elitist the dominance postulate $P 3$.

However, the fact is that each of these strategies is well-defined and has a coherent motivation (and many other such examples could also be constructed). To brand them irrational seems to me itself irrational dogma. Even the most contentious case, the rivalrous future self elitist, has a coherent, if ungenerous, philosophy of life in the multiverse and a rational strategy for implementing it. Note too that some of these strategies have arguable theoretical advantages over the mean utilitarian strategy. For instance, one can be an $x$-percentile utilitarian, or a future self democrat (if they are purely self-concerned, in the sense that each future self's preferences among options are completely determined by the implications for its own welfare), without having to quantify the utility of the possible outcomes: one needs only a preference ordering. This is arguably advantageous, since even if one accepts Savage's postulates [41] and, hence, the conclusion that one's preferences must be defined by some utility function, it may be difficult or even impractical to compute the relevant function for general outcomes, and yet relatively easy to identify preferences among any finite list of outcomes.

In short, Greaves-Myrvold's postulates only express in more abstract form a preference for being a mean utilitarian - i.e., for one possible choice among many. Their postulates are plausible possible prescriptions for rational behaviour when considering the welfare of a population of future selves, but also logically inconsistent with other plausible prescriptions. This shouldn't come as a complete surprise: after all, Arrow's celebrated impossibility theorem [43] taught us that plausible decision theoretic principles for populations may be inconsistent.

Granted, the one-world counterparts of some of these strategies may look peculiar. But one can consistently accept the many-worlds strategies as rational and reject their one-world counterparts. As Price [35] has persuasively argued, many-worlds agents can offer reasoned justifications for their strategies that aren't available to their one-world counterparts. The many-worlds future self elitist knows that his best possible future self will be an actual future self, while his one world counterpart doesn't. The many-worlds future self democrat knows that there really will be a population of future selves who have preferences among the betting choices, while her one world counterpart knows there won't be; and so on.

One could, of course, adopt a weaker position. One could take Greaves-Myrvold's and Wallace's accounts of rationality as simply suggesting a possible attitude one might adopt to life in an Everettian multiverse, an attitude defined by a set of rules which are consistent and have some pleasant mathematical features but which are not meant to constitute a dogma. On this liberal reading, Greaves-Myrvold's preferred strategy could be termed "rational", in the 
sense of being well-defined and internally consistent, without denying the existence of other equally rational strategies. The problem is that abandoning any claim of uniqueness also removes the purported connection between theoretical reasoning and empirical data, and this is disastrous for the programme of attempting to interpret Everettian quantum theory via decision theory. If Wallace's arguments are read as suggesting no more than that one can consistently adopt the Born rule if one pleases, it remains a mystery as to how and why we arrived at the Born rule empirically. If Greaves-Myrvold's arguments are read as merely suggesting a possible attitude one might choose to take about testing and confirming many-worlds theories, one's left to investigate how many other equally valid attitudes there might be, and whether they mightn't - disastrously - imply the confirmation of inconsistent theories from the same data.

\section{Rationality and feasibility}

Consider now a rather more complicated multiverse, $C B U_{4}$. Here, the universes are definitely being simulated by technologically advanced beings, and the inhabitants know it. They also know that, after the red button is pressed, there is a list of outcomes $i$, and that the list is indeterminately long (and possibly infinite). They know too that, for each $i$, some number of successor universes containing outcome $i$ will be created. They do not know the number of successors there will be of each type: these vary for each $i$, and vary each time the button is pressed, at the whim of the simulators. What they do know - because, let's say, the simulators have credibly promised them - is that numbers playing the role of additive weights, following certain rules, will be written inconspicuously into each simulated universe. Thus, if their universe has the number $x$ written in it, and the red button is pressed, and there are $n_{i}$ successor universes with outcome $i$, these successors will have numbers of the form $x q_{i}^{j}$ written into them, where the label $j$ runs from 1 to $n_{i}, q_{i}^{j} \geq 0$, and

$$
\sum_{j} q_{i}^{j}=p_{i} .
$$

Here the $p_{i}$ are known to be constants (i.e. they take the same value each time the button is pressed), with $p_{i} \geq 0$, and $\sum_{i} p_{i}=1$. The inhabitants know the values of a finite set of the $p_{i}$, those with index $i \in I$, whose sum $\sum_{i \in I} p_{i}<1$.

What the inhabitants would like to do - what they feel rationality would mandate they do if they could - is express betting/distribution preferences that value each successor universe equally. But they can't - they don't know how many successors will be created for any given $i$, nor do they know how long the list of possible outcomes $i$ is. Nor can they express betting/distribution preferences that value each outcome equally, regardless of the number of successor universes containing it - again, they don't know how long the list of possible outcomes is.

What they can do is express betting/distribution preferences, for bets on the known possible outcomes, treating the known values of $p_{i}$ as probability weights. Doing so is equivalent to treating a successor universe with the number $y$ written into it as having an importance proportional to $y$ - a rule which can be consistently applied, despite their ignorance about the number of successors of each type, because of equation (2). So, they have a consistent, feasible strategy available to them. Moreover, if they want to assign a measure of importance to each individual universe, and they want the importance they assign to the set of universes containing outcome $i$ to be independent of the number of such universes, this is the only available rule. Nonetheless, it doesn't seem to have a fundamental rational justification. The numbers written into the universes happen to follow convenient bookkeeping rules, but they have no significance: there is no fundamental reason to treat the numbers as a measure of importance of their universes.

From this, I think we should conclude two things, to be borne in mind when we come to consider Wallace's arguments. It can make perfect sense, in a multiverse theory, to say that there exists a rational optimal strategy that is inaccessible to the agents in that multiverse. Conversely, the fact that a strategy is available does not per se make it rationally compelling, even if it is the unique available strategy satisfying some pleasant consistency properties: rational compulsion also needs rational justification, which may or may not exist.

\section{WHY MANY-WORLDS THEORY CONFIRMATION DOESN'T WORK}

Everettian quantum theory is essentially useless, as a scientific theory, unless it can explain the data that confirm the validity of Copenhagen quantum theory within its domain - unless, for example, it can explain why we should expect to observe the Born rule to have been very well confirmed statistically. Evidently, Everettians cannot give an explanation that says that all observers in the multiverse will observe confirmation of the Born rule, or that very probably all observers will observe confirmation of the Born rule. On the contrary, many observers in an Everettian multiverse will definitely observe convincing disconfirmation of the Born rule. Nor can one look at Everettian quantum 
theory and conclude that any given observer in the multiverse will probably observe confirmation: the theory has no notion of standard probability available to even make sense of any such claim. And if the theory doesn't explain the data, the data don't support the theory.

There seems to be no good way around this, and if so, then that's the end of Everettian quantum theory as a serious contender: a theory with no predictive power should lose the scientific competition against theories that predict what we actually see. However, Greaves and Myrvold [24] have offered an attempt at a solution, by giving a general account purporting to explain why agents who take seriously the possibility of many-worlds theories can use observational data to confirm particular theories and refute others. Their account is illuminating, and raises some very interesting questions about many-worlds theories. Ultimately, though, it seems to me that it does not show, as claimed, the possibility of explaining our observations from a many-worlds theory and thus confirming one many-worlds theory against another. Rather, it highlights some apparently insuperable problems that prevent us from doing so. As Greaves and Myrvold's arguments are set out in detail elsewhere in this volume, in this discussion I will simply summarize the implications of their confirmation algorithm in toy models, and point out the problems that arise.

\section{A. The problem of inappropriate self-importance}

It suffices to consider very simple many-worlds theories, containing classical branching worlds in which the branches correspond to binary outcomes of definite experiments. Consider thus the weightless multiverse, a many-worlds theory of type $C B U_{1}$, in which the machine produces only two possible outcomes, writing 0 or 1 onto the tape. Recall that in $C B U_{1}$ there is no fact of the matter about weights attached to the branches containing 0 outcomes and 1 outcomes, although the inhabitants think there may be. This is the many-worlds analogue of an indeterministic one-world theory containing a sequence of binary experimental outcomes which are not only not determined but also not governed by any probabilistic law. Suppose now that the inhabitants begin a series of experiments in which they push the red button on the machine a large number, $N$, times, at regular intervals. Suppose too that the inhabitants believe (correctly) that this is a series of independent identical experiments, and moreover - this is not essential, but simplifies the discussion - believe this dogmatically: no pattern in the data will shake their faith. Suppose also that they believe (incorrectly) that their multiverse is governed by a many-worlds theory with unknown weights attached to 0 and 1 outcomes, identical in each trial, and seek to discover the (actually nonexistent) values of these weights by following Greaves-Myrvold's learning algorithm.

After $N$ trials, the multiverse contains $2^{N}$ branches, corresponding to all $N$ possible binary string outcomes. The inhabitants on a string with $p N$ zero and $(1-p) N$ one outcomes will, with a degree of confidence that tends towards one as $N$ gets large, tend to conclude that the weight $p$ is attached to zero outcome branches and weight $(1-p)$ is attached to one outcome branches. In other words, everyone, no matter what outcome string they see, tends towards complete confidence in the belief that the relative frequencies they observe represent the weights.

Let's consider further the perspective of inhabitants on a branch with $p N$ zero outcomes and $(1-p) N$ one outcomes. They do not have the delusion that all observed strings have the same relative frequency as theirs: they understand that, given the hypothesis that they live in a multiverse, every binary string, and hence every relative frequency, will have been observed by someone. So how do they conclude that the theory that the weights are $(p, 1-p)$ has nonetheless been confirmed? Because, following Greaves-Myrvold's reasoning, they have concluded that the weights measure the importance of the branches for theory confirmation. Since they believe they have learned that the weights are $(p, 1-p)$, they conclude that a branch with $r$ zeroes and $(N-r)$ ones has importance $p^{r}(1-p)^{N-r}$. Summing over all the branches with $p N$ zeroes and $(1-p) N$ ones, or very close to those frequencies, thus gives a set of total importance very close to 1 ; the remaining branches have total importance very close to 0 . So, on a set of branches that dominates the importance measure, the theory that the weights are (very close to) $(p, 1-p)$ is indeed correct. All is well! By definition, the important branches are the ones that matter for theory confirmation. The theory is indeed confirmed!

The problem, of course, is that this reasoning applies equally well for all the inhabitants, whatever relative frequency $p$ they see on their branch. All of them conclude that their relative frequencies represent (to very good approximation) the branching weights. All of them conclude that their own branches, together with those with identical or similar relative frequencies, are the important ones for theory confirmation. All of them thus happily conclude that their theories have been confirmed. And, recall, all of them are wrong: there are actually no branching weights.

\section{Comparison with the one-world case}

It's illuminating to compare the case of an inhabitant of the analogous one-world universe, in which pressing the red button produces either a 0 or a 1 on the tape but there is no law, either deterministic or probabilistic, governing 
these outcomes. After $N$ experiments in which he sees $p N$ zeroes and $(1-p) N$ ones, he tends towards confidence in the theory that zeroes have probability $p$ and ones have probability $(1-p)$.

Let us again restrict attention to theories — in this case probabilistic one-world theories — that dogmatically assume the experiments are identical and independent. Among such theories, the selected theory does indeed characterize, better than all its competitors, all the relevant data in the universe - i.e., all the outcomes of the $N$ experiments. Of course, further data could change that conclusion. But, so long as we consider only the relevant data, it's something of a puzzle to pin down whether it's wrong to adopt the theory pro tem, and if so precisely why. Is there a physically meaningful sense in which a universe that looks as though it contains data resulting from a sequence of independent identical coin tosses with a probability $p$ of outcome zero is distinct from one that does contain such data? And if so, how precisely should we characterize the distinction?

On the view of physical randomness discussed in section II the answer to the first question is no. In any case, however one answers the questions, it seems that any possible error here must be subtler than and distinct from the error highlighted above in the many-worlds case. In the many-worlds case, recall, all observers are aware that other observers in worlds with other data must exist, but each is led to construct a spurious measure of importance that favours their own observations against the others', and this leads to an obvious absurdity. In the one-world case, observers treat what actually happened as important, and ignore what didn't happen: this doesn't lead to the same difficulty.

\section{Numbers in the sky}

Consider next the decorative weight multiverse, a type $C B U_{2}$ variant of the weightless universe. This universe has a constant of nature fixed by the technologically advanced beings, a real number $p$, with $0<p<1$. As before, whenever the red button is pressed in a simulated universe, that universe is deleted, and successor universes with outcomes 0 and 1 written on the tape are initiated. This time, the technologically advanced beings also write the numbers $p$ and $(1-p)$ in an inaccessible part of the skies of the 0 and 1 successor universes, respectively. These numbers are visible to the inhabitants, but have no other physical significance.

There is thus a formal sense in which distinct weights are attached to the 0 and 1 branches. However, by hypothesis, these weights are decorative: there are no rational grounds for assigning them any fundamental physical meaning or any rôle in constraining rational actions. We can thus run through a discussion of theory confirmation precisely parallel to that for the weightless multiverse.

This illustrates again that the mere fact that Born weights are mathematically defined in Everettian quantum theory does not per se justify assigning them any role in theory confirmation. They could be merely decorative.

\section{B. Separating caring weights from theory confirmation}

To investigate further it's helpful to consider branching world models in which there are weights attached to the branches, in such a way that the weights could plausibly be regarded as important for making rational decisions. I want here to tell specific stories about the weights, in order to illustrate a crucial distinction between two possible definitions of importance.

\section{The replicating multiverse}

Consider first the replicating multiverse, a multiverse of type $C B U_{3}$ with a machine like the one above, in which the branches arise as the result of technologically advanced beings running simulations. Whenever the red button is pressed in a simulated universe, that universe is deleted, and successor universes with outcomes 0 and 1 written on the tape are initiated. Suppose, in this case, that each time, the beings create three identical simulations with outcome 0, and just one with outcome 1 . From the perspective of the inhabitants, there is no way to detect that outcomes 0 and 1 are being treated differently, and so they represent them in their theories with one branch each. In fact, though, given this representation, there is an at least arguably natural sense in which they ought to assign to the outcome 0 branch three times the importance of the outcome 1 branch: in other words, they ought to assign branch weights $\left(\frac{3}{4}, \frac{1}{4}\right)$.

They don't know this. But suppose, as before, that they believe that there are unknown weights attached to the branches, and follow the Greaves-Myrvold procedure for identifying those weights. What happens now? After $N$ runs of the experiment, there will actually be $4^{N}$ simulations - although in the inhabitants' theoretical representation, these are represented by $2^{N}$ branches. Of the $4^{N}$ simulations, almost all (for large $N$ ) will contain close to $\frac{3 N}{4}$ zeroes 
and $\frac{N}{4}$ ones. These simulations will contain inhabitants who, following Greaves-Myrvold, believe they have confirmed that the branch weights (in their own theoretical representation, which remember contains only $2^{N}$ branches) are very close to $\left(\frac{3}{4}, \frac{1}{4}\right)$. They believe too that the weights define an importance measure on the branches: a branch with $r$ zeroes and $(N-r)$ ones has importance (very close to) $\left(\frac{3}{4}\right)^{r}\left(\frac{1}{4}\right)^{N-r}$. They thus conclude that their weight assignment will be confirmed on a set of branches whose total importance is close to 1 .

Now, I think I can see how to run some, though not all, of an argument that supports this conclusion. The branch importance measure defined by inhabitants who find relative frequency $\frac{3}{4}$ of zeroes corresponds to the counting measure on simulations. If we could argue, for instance by appealing to symmetry, that each of the $4^{N}$ simulations is equally important, then this branch importance measure would indeed be justified. If we could also argue, perhaps using some form of anthropic reasoning, that there is an equal chance of finding oneself in any of the $4^{N}$ simulations, then the chance of finding oneself in a simulation in which one concludes that the branch weights are (very close to) $\left(\frac{3}{4}, \frac{1}{4}\right)$ would be very close to one. Turning that around, the theory that the branch weights are $\left(\frac{3}{4}, \frac{1}{4}\right)$ would then imply that, with high probability, one should expect to see relative frequency of zeroes close to $\frac{3}{4}$. There would indeed then to be a sense in which the branch weights define which subsets of the branches are important for theory confirmation.

It seems hard to make this argument rigorous. In particular, the notion of "chance of finding oneself" in a particular simulation doesn't seem easy to define properly. Still, we have an arguably natural measure on simulations, the counting measure, according to which most of the inhabitants will arrive at (close to) the right theory of branch weights. That might perhaps be progress.

\section{The qualia enhancing multiverse}

But consider now the qualia enhancing multiverse, again a multiverse with the same type of machine, in which the branches arise in the way we've previously considered, as the result of technologically advanced beings running simulations. Whenever the red button is pressed in a simulated universe, that universe is deleted, and successor universes with outcomes 0 and 1 written on the tape are initiated. This time, though, the beings create just one simulation with outcome 0 , and one with outcome 1 , but devise their simulations so that the qualia - the mental sensations - of the inhabitants in the outcome 0 simulation are three times as intense. As before, from the perspective of the inhabitants, there is no way to detect that outcomes 0 and 1 are being treated differently, and so they represent them in their theories with one branch each.

There is, again, an arguably natural sense in which they ought - if they were aware of the rules of their multiverse to assign to the outcome 0 branch three times the importance of the outcome 1 branch: in other words, they ought to assign branch weights $\left(\frac{3}{4}, \frac{1}{4}\right)$. Recall, pleasure and pain in outcome 0 branches have tripled in intensity. The welfare of successors on outcome 0 branches is felt more intensely, and in that sense it matters more.

Let me deal here with three possible objections:

(a) It might be argued that qualia enhancement should be analysed differently, as an example of an unannounced alteration in utility functions: the actual payoff of winning a bet with outcome 0 is three times the expected payoff, since the inhabitants don't expect any qualia enhancement. Certainly it could be analysed in this way. But this reflects an arbitrary choice that always needs to be made in many-worlds theories. (Precisely the same argument could be made in the case of the replicating multiverse, for example.) The statement that one branch is $N$ times as important as another can always be recast as a statement that utilities on the first branch are rescaled by $N$ relative to those on the second. So, we can legitimately analyse qualia enhancement as an effect altering the relative importance of branches, and it's interesting to do so, as this lets us test general propositions about the confirmation of theories attaching importance to branches.

(b) The reader may not believe that there is a sensible account of experience involving qualia, or that intensifying qualia makes any sense. Never mind. It's just a useful device to make a point about branch measures. It could be formulated in another way: we could suppose that the simulators arrange that all bets have payoffs with three times the expected utility on outcome 0, while erasing the relevant bits of the inhabitants' memories so that they're not aware that the payoff tripled.

(c) One might also worry that inhabitants in an outcome 0 branch would notice that the intensity of their qualia has just tripled. For the sake of the argument, we must assume not. Insofar as the notion of qualia enhancement makes sense, this seems reasonable: their memories will triple in intensity along with everything else.

Suppose, once again, that the inhabitants believe that there are unknown weights attached to the branches, and follow the Greaves-Myrvold procedure for identifying those weights. What happens now? After $N$ runs of the experiment, there will be $2^{N}$ simulations - now correctly represented by $2^{N}$ branches in the inhabitants' many-worlds 
theory. The simulations will contain inhabitants who, following Greaves-Myrvold, believe they have confirmed that the branch weights are very close to $(p, 1-p)$, because their observed relative frequency is $p=r / N$, for each $r$ in the range $0 \leq r \leq N$. They believe that the weights define an importance measure on the branches: a branch with $r$ zeroes and $(N-r)$ ones has importance (very close to) $(p)^{r}(1-p)^{N-r}$. They thus conclude that their weight assignment will be confirmed on a set of branches whose total importance is close to 1 . Now, in one sense, the inhabitants whose observed relative frequency $p=3 / 4$ are a special case. Their inferred importance measure equals the natural importance measure defined by qualia intensity. And if we weight the branches by this importance measure, it is the case, by the same calculation as before, that, on a set of branches with total measure close to one, the inhabitants end up with (very close to) the "right" branch weights, $\left(\frac{3}{4}, \frac{1}{4}\right)$.

But wait! If we count the simulations, the inhabitants who arrive at weights $\left(\frac{3}{4}, \frac{1}{4}\right)$ are a tiny minority. Almost everyone arrives at the wrong branch weights - and, as in our earlier example, almost everyone arrives at a measure of importance according to which branches with (very close to) their observed relative frequency are the important ones. By the natural simulation counting measure, theory confirmation has spectacularly failed.

What these last two examples show is that there are two distinct senses, which Greaves-Myrvold and Wallace fail to separate, in which a branch weight might possibly be said to be a measure of importance. It could be said to be a "caring measure", if there is some reason to care differently about the welfare of successors on different branches. And it could be said to be, for want of a better term, an "explanatory counting measure", if there is some reason to think that we are likelier to find ourselves on some branches rather than others - or some other argument to show that a branching theory which predicts the observed relative frequencies (or other data) on a set of branches of high explanatory counting measure thereby explains them. What we've seen is that the first property doesn't necessarily imply the second, and it's the second that is needed for an adequate account of branching theory confirmation.

Couldn't a many-worlds theorist then simply postulate the existence of an explanatory counting measure? (And perhaps also postulate that a caring measure exists and equals the explanatory counting measure?)

A preliminary remark: even postulating a caring measure - which has been proposed [22] in the Everettian literature - already seems a very strange manoeuvre. Physical theories can certainly give reasons for rational agents to perform certain actions if they have certain goals. But what's envisaged here is a theory that by fiat imposes a constraint on rational behaviour. I'm not clear - and at least some Everettians (e.g. [13]) seem to share this worry - that this makes any sense, either as an idea about physics, or about rationality. 5.5 .

In any case, when it comes to postulating an explanatory counting measure, one should be clear: the proposal is that a many-worlds theory defines, by fiat, without any attempt at further justification, whose observations matter and whose may be neglected, when it comes to testing and confirming the theory. The theory defines its own - highly non-standard - criteria for deciding whether or not it is a scientific success.

One could play this sort of game, of course, even in one world. For example, Alice could define a theory that includes - as a postulate, with no further explanation - the principle that everyone who agrees with her observations and her theoretical interpretation is important for theory confirmation, and everyone else is negligible. She could then announce, after checking with the important people, that her theory is confirmed. This would be self-consistent, and maybe politically adept, but it wouldn't be science.

It's no more scientifically respectable to declare that we can, without further justification, confirm Everettian quantum theory by neglecting the observations made on selected low Born weight branches. A Pavlovian association of low Born weight with small probability - illegitimately carried over from one world quantum theory - may perhaps lend an aura of greater respectability. But in Everettian quantum theory the Born weight is simply a number attached to branches. It has no intrinsic relevance to theory confirmation, and unless we add further structure to the theory, we cannot justify assigning it any such role.

Note again the contrast here with the one-world case: one-world probabilists do not pick and choose which observations are to be used for theory generation or confirmation.

\section{Many-worlds confirmation: conclusion}

To explain how we could come to confirm Everettian many-worlds quantum theory it is not enough to note that we have Born weights to hand and so can automatically give them a confirmation-theoretic rôle. As the decorative weight multiverse illustrates, branch weights can be simply irrelevant to theory formation and confirmation.

Nor can Wallace's arguments for treating the Born weights as a caring measure suffice, even if we take Wallace's result at face value. As the qualia enhancing multiverse illustrates, a caring measure is not necessarily an explanatory counting measure.

Thus, the most sympathetic (though unauthorised) translation of Greaves-Myrvold's account of many-worlds and confirmation that I can find requires us to add structure that justifies the existence of an explanatory counting measure. This requires interpreting Everettian quantum theory, along with competing many-worlds theories theories, 
as modelled by versions of the replicating multiverse, with branches constantly being deleted, and successor branches created. We need to postulate that the number of simulations or realisations of a given branch at a given time is proportional to the branch weight, and to assume that it is rational to treat all realisations as equally valuable. We need also to postulate something like an anthropic principle that tells us that, in some sense that needs to be properly defined, the chance of finding ourselves in one of a given class of realisations at a given time is proportional to the number of realisations in the class.

This, if it could be made rigorous, would suggest something resembling the objectively determinist "momentary minds" version of Albert-Loewer's many-minds interpretation [14 16], in which the minds exist only instantaneously, with no continuous identity extending over time. This isn't a picture I find easy to take seriously. As I read them, none of the Everettian contributors to the present volume would wish to defend this account - and yet it seems very closely aligned with some of their intuitions. Let me close here by inviting readers to see if they can find a better way of rigorously justifying Greaves' gloss: 23]

But since we have a measure over our successors, we can, if we find it intuitive, talk of 'how much successor' sees spin-up. I have a preference for my spin-down successor to receive chocolate, rather than my spin-up successor, because there is more of the former; more of my future lies that way. Thus, I think, Lockwood's (1996) talk of a 'superpositional dimension', and/or Vaidman's $(1998,2001)$ suggestion that we speak of the amplitude-squared measure as a 'measure of existence', are somewhat appropriate (although we are not to regard lower-weight successors as less real, for being real is an all-or-nothing affair - we should say instead that there is less of them).

\section{FUZZINESS, RATIONALITY AND DECISION THEORY IN MANY WORLDS}

Two of the most interesting recent developments in the Everettian literature, in my view, have been the attempt to argue for an intrinsically fuzzy emergent quasiclassical ontology [19 and (as already discussed) the attempt to reinterpret Born weights via a many-worlds version of decision theory [20]. Interesting, but flawed - each project has deep problems, and they appear to be based on inconsistent premises.

\section{A. Fuzziness and its limitations}

Granted, as Wallace 19] notes, viable higher level scientific theories can and do, indeed, supervene on more fundamental theories. Objects in those theories need not have any unique and precise definition in terms of fundamental concepts: there is, indeed, no unique, natural, precise, chemical characterisation of a tiger.

Nonetheless, there is a very strong reason for seeking [45] a precise mathematical formulation of the intuition that many branching worlds emerge from unitary quantum theory - or else a precise mathematical formulation of some other structure consistent with Everettian ideas - namely, that it is not at all clear that, without such a formulation, we have a well-defined scientific theory to discuss. (This, it seems to me, is why both Everettians [8, 11] and critics [15, 16] have often attempted to find mathematical structures that might explain the notion of branching.) The alternative strategy, proposed by Wallace [19], of trying to interpret the implications of a fundamentally mathematical theory in terms of higher level fuzzily defined constructs carries a very obvious danger - namely, a retreat into vagueness and hand-waving on points where precision really is required. It's hard to run a serious argument (pro or con), let alone prove a rigorous theorem, if one doesn't, in the end, know quite what one's talking about.

\section{B. Fuzzy minds}

A case in point is Wallace's appeal to functionalist intuitions in trying to give an account of the mind states of agents in Everettian quantum theory. Readers are, I think, owed a much more precise explanation of what, actually, is supposed to follow from this, since some rather crucial points appear to turn on unspecified details.

For instance, on this account, do distinct mind states necessarily correspond to orthogonal quantum states? If so, wouldn't this account necessarily supply us with a preferred orthogonal decomposition of the unitarily evolving quantum state? And wouldn't this, pace Wallace 19], allow a precise definition of a relevant branching structure after all?

Wallace places great emphasis on the lack of a unique natural definition of a quasiclassical branch, and hence the impossibility of agents formulating a rational strategy based on counting distinct future branches. But it's at least as relevant to examine whether our account of mind states supplies a natural definition of a future self, and whether 
it might be possible for agents to formulate a rational strategy based on counting distinct future selves? Can't an agent identify successor selves as distinct if and only if they have distinct mind states, ascribe to distinct successors a branching history corresponding to that recorded in their memories, and use those data to define a rational strategy for taking account of their welfare? (These points are pursued further in appendix $\mathrm{A}$ )

On the other hand, if non-orthogonal quantum states could correspond to distinct mind states, how would we even begin to connect quantum theory with even the appearance of probabilities? Quantum theory gives no general rule to calculate a probability of a transition from an unknown state belonging to one fuzzily defined set of states (corresponding to mind state A) to an unknown state belonging to another (corresponding to mind state B). But that's what we'd need to calculate, in principle, in order to obtain a number corresponding to the apparent probability of arriving at state B when starting in state A. Maybe one could cook up such a rule, and then explain how the Born rule emerges as an approximation under suitable circumstances - but it's not obvious how, and this would certainly be going beyond quantum theory as presently understood.

Both options thus lead to serious, perhaps insuperable, difficulties.

\section{Can precise preferences arise in a fuzzy ontology?}

Another very basic worry about Wallace's programme is its equivocation over mathematical rigour. Everything in Wallace's ontology that's relevant to rational decisions - including agents, the quasiclassical branches they inhabit, the branch states, and the branch Born weights, and the distinction between micro-states and macro-states - is intrinsically fuzzily defined [19]. There is, on Wallace's account, no precise fact of the matter about the different quasiclassical states that would result after a bet on a quantum experiment, nor about the Born weights of the branches corresponding to those quasiclassical states. And this isn't merely because quantum theory doesn't supply a unique natural definition of elementary branches and branching events: the total Born weight of all the quasiclassical branches describing a spin-up outcome of a Stern-Gerlach experiment isn't precisely defined either.

Now, to be sure, the total weight is supposed to be approximately defined. We are supposed, on Wallace's account, to be able to say that it's in a range of the form $R=(p-\epsilon, p+\epsilon)$, where $\epsilon$ is very small, and $p$ thus represents an approximate total Born weight. [56] But we're not supposed to be able, on this account, to reduce $\epsilon$ to zero: below some level of precision, it becomes unavoidably arbitrary, just a matter of taste in your choice of branch definition, whether you take the total weight as $p_{1} \in R$ or $p_{2} \in R$.

And yet, Wallace's decision theoretic programme postulates that each rational agent should have a precisely specified and complete preference ordering among a very large class of possible unitary maps that produce different possible future global states. Where could such a preference ordering possibly come from? The ordering is supposed to be agent-dependent. Physics doesn't equip rational agents with some personal preference ordering on global states: they have to arrive at their preferences by introspection and reasoning. If one accepts Wallace's conclusions, the only ultimately relevant quantities are branch weights and the agent's personal utilities for macrostates (whose existence is supposed to follow given the preference ordering axioms). But even a super-agent who finds they can calculate the former and can identify the latter by pure introspection would find these quantities only fuzzily defined - so that, in comparing some pairs $\left(U_{1}, U_{2}\right)$ of actions on a given state $|\psi\rangle$, however hard they try and however carefully they analyse the alternatives, they wouldn't be able to identify a reliable preference, not because the resulting global states are precisely equivalent, but because their difference is fuzzily ambiguous. On some views, $U_{1}|\psi\rangle$ would seem very slightly preferable; on others, $U_{2}|\psi\rangle$ would. In Wallace's notation [19] for preference orderings, neither $U_{1} \succeq_{\psi} U_{2}$ nor $U_{2} \succeq_{\psi} U_{1}$ would hold in all ways of looking at the situation. Nor does it seem legitimate to postulate that $U_{1} \sim_{\psi} U_{2}$ must hold in such cases. One can imagine the possibility of a sequence $\left(U_{1}, \ldots, U_{n}\right)$ such that no preference can reliably be identified between $U_{i}|\psi\rangle$ and $U_{i+1}|\psi\rangle$, for $i=1, \ldots,(n-1)$, but nonetheless setting $U_{i} \sim_{\psi} U_{i+1}$ violates transitivity, since $U_{1} \succ_{\psi} U_{n}$ does hold no matter what view the agent adopts of the fuzzy facts.

We are not, in any case, super-agents, and can only read Wallace's arguments as prescriptions for ideal rationality rather than descriptions of our real-world behaviour. None of us in fact has a complete and precise preference ordering among the relevant unitaries. Wallace, in effect, is telling us that we should ideally adjust our reasoning and their behaviour so as to be consistent with some complete preference ordering. But how? There is no natural algorithm available: any choice will involve uncountably many arbitrary decisions on pairs of preferences. 44] And why? Given that no choice of ordering will have any intelligible justification, even after the entire analysis is complete, how can there be a rational compulsion to make some choice (even if, counterfactually, it were practical)?

Here, it seems to me Wallace's prescription runs into essentially the same difficulties that he identifies in other ways of thinking about Everettian branching. One could, in principle, find some (perhaps ad hoc) prescription defining a branching structure for the unitarily evolving state vector, and one could then use this structure to define a rational Born-rule-independent strategy based on branch counting. Wallace accepts that such a strategy is not logically inconsistent, but argues that it is likely to be difficult to implement in practice (because defining a precise branching 
structure is difficult) and hard to justify in principle (because the definition seems to require ad hoc choices). Both objections apply - arguably with at least equal force - to the Wallace programme.

This also reinforces the point that the case for Wallacean rationality cannot possibly rely on the lack of any practical alternative strategy. A very practical alternative is to follow whatever combination of instinct and reasoning evolution provided us before we became aware of Everettian quantum theory. Altering that strategy so as to comply rigorously with Wallace's axioms isn't practical; even coming close to doing so may not be. To be persuaded that we ought to try, we would need to be rationally persuaded not only that we should ideally be Wallaceans, but also that there is a practical method which allows us to become closer to being Wallaceans, and that we will be better off if we employ this method. [57]

In short, given Wallace's account of a fuzzy ontology, there seems a strong reason to doubt Wallace's most basic postulate of rationality, $R 1$, which states that rational agents have a complete (or connected) preference ordering on the unitary operations available to them at any state $|\psi\rangle$. No actual agent in a fuzzy Everettian ontology will ever be able to arrive at such an ordering in practice. Moreover, even if they had infinite computational power, fixing an ordering would require making a very complicated ad hoc choice which can have no complete rational justification. Yet without $R 1$, the purported derivation of the Born rule 20] fails at the first step.

It's not clear to me that there is any fix for this, but let me comment briefly on two possible responses.

First, one might perhaps try weakening the postulate $R 1$ to suggest that agents have, or should aspire to have, a preference ordering that approximates a complete ordering, in the hope of then proving that their policy should approximate Born-weighted mean utilitarianism. One problem with this is that one would need first to find and justify a suitable definition of approximation applied to preferences between pairs of unitary operations. As these are unquantified binary relations, it doesn't seem obvious that any suitable definition exists.

Second, one might consider the desperate resort of postulating a total ordering as part of the physical theory. But even that surely isn't available here. The orderings, recall, are agent-dependent, and even the most postulate-happy Everettian would surely recoil from requiring that fundamental physical laws specify independently, agent by agent, the preferences of every agent instantiated in nature.

Trying to formulate a rigorous decision theory for preferences in a fuzzy ontology may thus be rather like trying to build a skyscraper on mud.

\section{Circularity of the Wallace programme?}

Zurek [26] flags another worry about the logical relation between the two parts [19, 20] of Wallace's programme, namely an apparent circularity. Wallace envisages a fuzzy quasiclassical ontology arising as the result of mathematical regularities observable within components of the unitarily evolving universal wave function. These regularities are supposed, in a realistic cosmological model, to arise through the decoherence of classical variables and to be defined by what Gell-Mann and Hartle term a quasiclassical domain [25], in which, for example, operators approximately quantifying local mass densities approximately follow classical equations of motion with probability close to one. Here the probability for a history defined by a sequence of operators $P_{1}\left(t_{1}\right), \ldots, P_{n}\left(t_{n}\right)$ is given by the decoherence functional

$$
\operatorname{Tr}\left(P_{n}\left(t_{n}\right) \ldots P_{1}\left(t_{1}\right) \rho_{\text {initial }} P_{1}\left(t_{1}\right) \ldots P_{n}\left(t_{n}\right)\right) .
$$

In other words, the ontology is defined by applying the Born rule. Even if one could show, as Wallace claims, that agents defined within that ontology are rationally justified in using the Born rule as a calculus for decisions, it would seem incorrect to portray this argument as a derivation of the Born rule within Everettian quantum theory. Wallace's argument should rather be understood as attempting to show something weaker: that the Born rule re-emerges as output (albeit, to be fair, in an interesting and non-obvious way) if assumed as input. Even if correct, this would leave open the possibility that there are many different consistent and essentially inequivalent ways of defining ontologies that include distinct types of agents for whom different rational decision calculi can be established. It would thus fail to explain whether and (if so) why our own decision calculus should be based on the Born rule. It would also leave open the questions as to whether and (if so) how agents in some consistently defined Everettian ontology can arrive at the rational decision calculus appropriate to their ontology.

\section{E. Problems with Born-weighted mean utilitarianism}

Wallace 20], developing earlier ideas of Deutsch [12], partly in response to criticisms (e.g. Ref. 37]) of the latter, then goes on to argue that from a few simple and purportedly natural axioms we can prove that rational agents who 
believe themselves to be in a universe described by many-worlds quantum theory are rationally required to (a) have a utility function that quantifies the value they assign to possible future quasiclassical events, (b) act so as to maximise their Born-weighted mean utility.

As we just saw, Wallace's first postulate, $R 1$, seems to run into a fundamental obstacle, since neither Born weights nor quasiclassical histories (and thus their utility) are precisely defined in his ontology, and without $R 1$ the decision theoretic argument, which, inter alia, implies the existence of a utility function, fails. Moreover, even for an agent who has a utility function applicable to all relevant quasiclassical histories, the strategy of maximising Born-weighted mean utility is not well-defined. For a real world agent in state $\psi$ there will generally be available unitaries $U_{1}$ and $U_{2}$ for which it's a matter of arbitrary definitional choice whether $U_{1}$ or $U_{2}$ produces higher Born-weighted mean utility.

There's a further practical problem, which isn't apparent in simple models of many-worlds experiments but is a serious worry in realistic applications. To be a rigorous Born-weight mean utilitarian in the real world, one must allow for the possibility of small Born weight branches with extreme negative or positive utility. The mean Born-weighted utility of a bet that, with Born weight close to 1, involves small utility gains or losses, is radically altered if it also creates a $10^{-25}$ Born weight branch of utility $-10^{30}$. Now, the Deutsch-Wallace-Savage arguments imply no bounds on agents' utility functions. It seems unlikely that any a priori argument can supply one, since pure rationality imposes no bound on utility functions - and in practice, for example, there seems to be no generally agreed lower bound on the utility cost assigned to the destruction of the Earth or similar catastrophes [38]. A rigorous real world Born-weight mean utility calculation thus typically requires very careful analysis of small weight branches. In fact, even ensuring that the sum defining the mean utility converges requires careful analysis of small weight branches: consider, for example, the possibility of a set of branches of weight $2^{-n}$ and utility $-3^{n}$ for all integers $n \geq N$.

Practically speaking, the best that real world agents are likely to be able to do is first simplify their model, by excluding events below some weight threshold, and then estimate a Born-weighted mean utility within that model with no assurance that the estimated mean utility is close to the true mean utility (if indeed the latter exists). [58] This needs emphasising, since much of Wallace's case against alternative rational strategies is based on the claim that they are ill-defined or impractical or both. Actually, as we will see, alternative strategies can sometimes be rather better defined and more practical than Born-weight mean utilitarianism.

\section{F. Everettian many-worlds rationality reconsidered}

\section{General remarks on life in a multiverse}

It seems prima facie surprising to claim that mathematical analysis could show that Born-weight mean utilitarianism, or any other strategy, is the unique rational way of optimizing the welfare of one's own, and other people's, many future selves in a multiverse. After all, human parents are faced with the not entirely disanalogous question of how to take into account the welfare of their genetic descendants in (most of us assume) a single world, and it's a notoriously complex problem. People generally care not only about their descendants' present welfare, but also about their expected future welfare after our death. They can, and sometimes do, frame guiding rules of thumb to arbitrate between competing claims on their resources - for instance, to divide their estate equally among their children, or to divide it according to their need. They take into account their children's relationships with one another, with others, and with society. They tend to care about immediate descendants more than distant ones, in a way that generally follows no well-defined formula. Evolutionarily developed instincts also impel a more general concern for our genes and those of the species. This concern probably cannot be precisely codified, but we can often find principles with which they are roughly aligned and which roughly characterise the behaviour they motivate. For instance, some species' instinctive behaviour might be roughly modelled as aiming to maximise an individual's expected number of descendants after $10^{2}$ years. Some humanists' aims might be modelled as aiming to maximise the survival probability of the human race (and its genetic successors) over the next $10^{9}$ years.

Some of these principles require impossible calculations to implement precisely, but can nonetheless legitimately be regarded as rational aims. If we adopt them, we commit ourselves to trying to satisfy them as best we can. In general, they imply conflicting courses of action. No one, I think, would seriously claim that any one of them is uniquely rationally preferable to all the others. We just make decisions as best we can, imperfectly guided by logic, sometimes perhaps trying our best to optimise quantities we know we cannot properly calculate. And we always did: before we were capable of rational reflection, evolution equipped us to muddle through, sometimes following one rule of thumb, sometimes another. That's life. Why should we expect evolution or rationality to have equipped us any better when faced with the bewilderingly underdetermined imperative to care about our and everyone else's quantum descendants in a hypothetical multiverse? 


\section{Alternative Born-weight-sensitive strategies}

Suppose, for the sake of the discussion, that we can somehow ignore the fuzziness of the ontology. Suppose that we have an agent faced with a finite number of choices $j$, each of which will create quasiclassical branches (although not a unique quasiclassical branching structure) with well-defined utilities $U_{i}^{j}$, in such a way that the set $S_{i}^{j}$ of branches with the same utility $U_{i}^{j}$ has a well-defined total Born weight $p_{i}^{j}$, and that the sums $\mu^{j}=\sum_{i} p_{i}^{j} U_{i}^{j}$ are finite. 59.

Consider again some of the strategies listed in Sec. ЩIB The $x$-percentile utilitarian, for $0<x<100$, always has a well-defined strategy, as does the future self democrat. The future self elitist and Price-Rawlsian's strategies are defined provided that $\max _{j} \sup _{i}\left(U_{i}^{j}\right)$ and $\max _{j} \inf _{i}\left(U_{i}^{j}\right)$, respectively, are defined. These will always hold true if the indexing set $I \ni i$ is finite. They need not hold true if the branch utilities are unbounded above or below (possibilities which are not usually considered by Everettians, and which perhaps might be excluded by assumption, but possibilities nonetheless).

As a practical matter, unless low Born weight extreme utility branches can be excluded, the future self elitist and Price-Rawlsian may have difficulty optimising their strategies, even if an optimal strategy exists, since calculating $\sup _{i}\left(U_{i}^{j}\right)$ or $\inf _{i}\left(U_{i}^{j}\right)$ requires analysing low Born weight branches that realise, or converge towards, the extreme utility values. This is also be a problem - which may be easier or harder, depending on the details - for the mean utilitarian. Generically, it should not be a significant problem for the $x$-percentile utilitarian (for most $x$, say $1<x<99$ ), assuming the utility function is generically well-behaved over the range, since the utility at the $x$-th percentile is then relatively insensitive to small perturbations of $x$, and so the calculation is relatively insensitive to the details of low Born weight extreme utility branches. It should also generally not be a problem for a purely self-concerned future self democrat, who would generally hope to be able to attain a majority decision without counting the votes from low Born weight extreme utility branches. [60]

\section{Some other strategies}

The Gell-Mann-Hartle aesthete fixes a particularly pretty quasiclassical consistent set $S$, which she uses to define a way of counting branches containing her future selves. 61 Her quantum ontology is Everettian: she agrees that her selected set has no fundamental physical significance. However, she thinks one needs some way of weighting future selves and that this one is as rationally defensible as Born-rule-weighting or any other, and more aesthetically pleasing.

The value teleologist fixes a particular cosmological final density matrix $\rho_{f}$, whose spectrum does not include zero. In considering whether or not to accept a generalized bet on a quantum experiment, or indeed making any decision dependent on a quantum event, he uses pre- and post-selection, with some standard theory of the initial cosmological conditions defining the initial state $\rho_{i}$, and with $\rho_{f}$ defning the final state, in order to calculate the probabilities of the future worlds corresponding to the possible outcomes. [46, 47] He bets as if these were the actual probabilities. This is not because he believes they are - he believes in deterministic unitary quantum mechanics and so doesn't think probabilities are fundamental, and in any case his physical theory is a standard cosmological theory with initial state $\rho_{i}$ and no post-selection on $\rho_{f}$. However, for aesthetic or existential reasons, his interest in future events is conditional on the chosen final state post-selection.

\section{Wallace's rationality postulates}

We noted already that branch weight, branch macrostate, branch microstate, and reward are all only fuzzily defined in Wallace's ontology [19], and that this gives strong reason to doubt Wallace's ordering axiom $R 1$. It casts doubt too on whether the availability axioms $A 3-A 5$ and the rationality axioms $R 3, R 5$ even have a precise definition.

Wallace's diachronic consistency axiom, $R 2$, is violated by the $x$-percentile utilitarian strategy, among others. Now, to be fair, one can find examples where the two conflict which illustrate some motivation for diachronic consistency. Consider the possibility of being offered $N$ dollars per unit time to stand in a radiation field, with a risk $p$ of lethality per unit time. An $x$-percentile utilitarian who considers this offer will generally find their response depends on the timescale over which they regard their decisions as binding: it could seem a good offer considered as valid for the next second, and then good again for each successive second, but a bad offer if they have to make a single decision about whether to accept for the next hour.

Yet, even in this rather unusual example, the motivation for $x$-percentile utilitarians is still clear when $x$ is close to 0 or 100, and their actual strategy is intended as a practical approximation to their ideal strategy of Price-Rawlsianism or future self elitism. The Price-Rawlsian will decline unless the total risk is zero; the future self elitist will accept unless the survival probability is zero. Note too that even here $x$-percentile utilitarianism is a well-defined strategy 
once a timescale for decisions is fixed. In the more normal circumstance of separated discrete decisions, $x$-percentile utilitarianism seems both rationally defensible and practical, which suggests that the diachronic consistency axiom is less rationally compelling than Wallace argues.

Another reason to doubt $R 2$, it seems to me, is that, pace Wallace's comment 20] -

"In the presence of widespread, generic violation of diachronic consistency, agency in the Everett universe is not possible at all."

- diachronic consistency actually is, strictly speaking, generically violated in real world decisions. A Savagean or Wallacean rational agent, recall, has to be equipped with a utility function as well as a probability measure for outcomes. Rationality is silent on the precise form of the utility function. If we have one, it reflects our current values. These generally change over time, as we do, partly as a result of decisions we have previously taken, whose outcomes affect us in ways we cannot reliably predict beforehand: our own natures are too complex and too opaque to us, and we also change in response to our environment, which is also complex and unpredictable. The best it seems to me that one might hope to say of diachronic consistency in real world decisions is that pretty often, in the short term, it approximately holds - which clearly isn't a strong enough assumption to prove an interesting decision theoretic representation theorem.

Elga's proposal that Everettians might have a rational preference for future self diversity [39] also seems pertinent here, as does the case for rationally preferring future society diversity. (Why not exploit the scope for political compromise by causing society to evolve in different ways along different branches?) In both cases, it seems to me, contra Wallace 39], diachronic consistency can be rationally violated. I can consistently believe now that it's a good thing that the global state should include future copies of me as a king and a beggar, while knowing that, if I ever find myself a beggar, I would strive to become a king if I could. 62 From the perspective of my future beggar self, the unpleasantness of finding that he is the beggar outweighs the satisfaction of knowing that diversity was achieved. From my present perspective, the prospect of diversity nonetheless remains appealing.

As Wallace himself notes earlier in his discussion [40]:

... to make a copy of myself and send him off to do a dangerous or disagreeable task - and ... to take actions designed to prevent him shirking that task ... is not irrational.

Indeed - and this remains true if the task, for which I have a strong present desire, is to ensure future self diversity. The fact that my future selves will never interact makes no difference to the rational justification.

Turning briefly to other postulates:

(R3) Microstate indifference, $R 3$, can be violated by value teleologist strategies, among others.

(R4) Continuity, $R 4$, is violated by $x$-percentile utilitarian strategies, among others. 63.

(R5) Branching indifference, R5, is violated by Gell-Mann-Hartle aesthete strategies, among others.

\section{Summary}

Wallace argues that strategies other than mean utilitarianism turn out, on closer inspection, either to be not rigorously defined, completely impractical, or to violate criteria such as diachronic consistency that allegedly define the very essence of rationality. The last two claims - impracticality and violation of rational essentials - surely require mathematical underpinning and justification, if they are to have any possible relevance to what is presented as a rigorous mathematical argument. For example, an account of practicality needs some complexity criteria for rational agent computations: one could then at least discuss the empirical justification for the proposed criteria and whether and when they actually distinguish mean utilitarianism from other strategies. Similarly, if one accepts that diachronic consistency is generically violated and sometimes grossly violated in the real world, an account of its role in decisions needs to quantify and compare the degree of violation implied by different strategies in different circumstances. At present, though, the arguments for diachronic consistency and those concerning practicality rest only on very debatable verbal intuitions.

As for lack of rigorous definition, there seems to be a danger of a double standard, whereby the fuzziness of the ontology is used to point out difficulties for alternative strategies (though in fact it also causes difficulties for mean utilitarianism), while the arguments for mean utilitarianism are justified in the context of toy models in which a precise definition of a branching structure can be found (in which case many strategies other than mean utilitarianism can be precisely defined). The case has not been made that mean utilitarianism is well-defined or practical in Wallace's fuzzy Everettian ontology, in which the mean utility of a strategy can at best only be fuzzily defined. One can imagine examples in which it either fails to be finite or is impractical to estimate - and it seems hard to exclude the possibility 
that these features often apply in the real world. One can also easily construct examples in which other strategies are easier either to approximate or to implement precisely.

Wallace's rationality postulates, likewise, are hard to motivate in Wallace's fuzzy Everettian ontology, where they are ultimately intended to apply, but where they are difficult, perhaps impossible, to define precisely. They generally appear, in any case, possible but uncompelling guides for rational agents. Where defined and practical, mean utilitarianism is certainly a rationally defensible strategy, with some mathematically convenient properties. But, like other critics [34, 35], I am far from persuaded that, if I were an Everettian, I should or would be a Born-weighted mean utilitarian.

\section{AGAINST SUBJECTIVE UNCERTAINTY}

One of the stranger claims in the recent Everettian literature is the suggestion, first made by Saunders [13, 48 50], that Everettian quantum theory, although deterministic, nonetheless has a natural probabilistic interpretation that can be found not by amending the theory or by adding further postulates, but simply by - somehow - analysing the experience and linguistic usages of agents, that is, creatures like ourselves, in an Everettian universe. In support of this claim are offered highly technical and controversial arguments concerning the philosophy of language. It seems to me simply a mistake, an exercise in wish fulfilment, to think that anything of significance to fundamental physics could turn on such questions, as though waving the magic wand of linguistic philosophy over a unitarily evolving state vector could somehow conjure up a probability measure and a sample space. 64]

Consider Wallace's succinct summary[50] of the argument:

"[The argument for subjective uncertainty] may be summarised as follows: in ordinary, non-branching situations, the fact that I expect to become my future self supervenes on the fact that my future self has the right causal and structural relations to my current self so as to count as my future self. What, then, should I expect when I have two or more such future selves? There are only three possibilities:

1. I should expect abnormality: some experience which is unlike normal human experience (for instance, I might expect somehow to become both future selves).

2. I should expect to become one or the other future self.

3. I should expect nothing: that is, oblivion.

Of these, (3) seems absurd: the existence of either future self would guarantee my future existence, so how can the existence of more such selves be treated as death? (1) is at least coherent - we could imagine some telepathic link between the two selves. However, on any remotely materialist account of the mind this link will have to supervene on some physical interaction between the two copies - an interaction which is not in fact present. This leaves (2) as the only option, and in the absence of some strong criterion as to which copy to regard as "really" me, I will have to treat the question of which future self I become as (subjectively) indeterministic."

This is a false trichotomy. Consider an (obviously simplified) Everettian description of an experiment in which an agent Alice, initially in brain state $|0\rangle_{A}$, observes a system in a quantum superposition $\sum_{i=1}^{2} c_{i}|i\rangle_{S}$, where $|1\rangle_{S}$ and $|2\rangle_{S}$ correspond, say, to the up and down states of a spin $1 / 2$ particle, and becomes entangled:

$$
|0\rangle_{A} \sum_{i=1}^{2} c_{i}|i\rangle_{S} \rightarrow \sum_{i=1}^{2} c_{i}|i\rangle_{A}|i\rangle_{S}
$$

Here $|i\rangle_{A}$ is Alice's brain state after observing the system in state $i$, for $i=1,2$.

Now, as an aside, we actually should take possibility (3) seriously, for two reasons. First, our conclusions ought to be based on empirical evidence rather than prejudice. We do not know that Everettian quantum theory is actually correct; we do not have a good theory of how consciousness is attached to quantum states; we do not know that we or any other agents have ever been in a superposition of macroscopically distinct brain states. We thus do not know whether, if we were able to place an agent in such a superposition, they would experience anything — nor, if so, what. Second, there's a coherent view of Everettian quantum theory in which we are continually being replaced by multiple copies of future selves. On this view, even if we assume that superposed selves have individual experiences, we will experience nothing in future (though our various future selves will).

The more immediately pertinent point, though, is that if we do take Everettian quantum theory seriously, it says, indeed, that Alice becomes entangled in a macroscopic superposition. A coherent way of describing this, which respects the link between brain states and mind states, is that just as materially she becomes several future selves, her 
mind becomes several disjoint, non-interacting future minds, with no telepathic link: i.e. option (1) without Wallace's misleading gloss.

The one description that seems obviously wrong, given the rules of the game Wallace sets out, is option (2): this really is an account of mind that supervenes on something not present in the physics, namely a probabilistic evolution law taking brain state $|0\rangle_{A}$ to one of the states $|i\rangle_{A}$.

The dangers of attaching some fuzzy theory of experience to Everettian quantum theory provoke two comments:

First, the fact that we don't have a good theory of mind, even in classical physics, doesn't give us a free pass to conclude anything we please. That way lies scientific ruin: any physical theory is consistent with any observations if we can bridge any discrepancy by tacking on arbitrary assumptions about the link between mind states and physics. We should, rather, be all the more cautious and tentative in offering any conclusion.

Second, the fact that at present no theory of mind can be expressed purely mathematically doesn't remove the obligation to strive to express one's ideas in mathematics as far as possible. Adorning Everettian quantum theory with extra assumptions expressed in words - for instance, as arguments in linguistic philosophy - without equations doesn't alter the fact that one's making extra assumptions: it merely makes them more vaguely expressed.

Consider 65] Saunders' exposition [13]:

Consider a simple example. Alice, we suppose, is about to perform a Stern-Gerlach experiment; she understands the structure of the apparatus and the state preparation device, and she is convinced EQM is true. In what sense does she learn, post-branching, something new? The answer is that each Alice, postbranching, learns something new (or is in a position to learn something new) - each will say something (namely, "I know the outcome is spin-up (respectively, spin-down), and not spin-down (respectively, spinup)") that Alice prior to branching cannot say. It is true that Alice, prior to branching, knows that this is what each successor will say - but still she herself cannot speak in this way. The implication of this line of thought is that, appearances notwithstanding, prior to branching Alice does not know everything there is to know. What is it she does not know? I say "appearances notwithstanding" for of course in one sense (we may suppose) Alice does know everything there is to know: she knows (we might as well assume) the entire corpus of impersonal, scientific knowledge. But what that does not tell her is just which person she is - or where she is located - in the wave-function of the universe.

But equation (4) suggests there is no meaning to this question before the experiment. 66 Nothing in the mathematics corresponds to "Alice, who will see spin up" or "Alice, who will see spin down". On the left we have "Alice, before the experiment"; on the right we have "Alice, who has seen spin up" and "Alice, who has seen spin down". If one wants to postulate an "Alice, who will see spin up", well, one can - but one should then include her in the mathematics. One could, for instance, start with a postulate of the form:

"(P) the probability that A's mind ends up believing that spin is up is $\left|c_{0}\right|^{2}$ and the probability that A's mind ends up believing that spin is down is $\left|c_{1}\right|^{2}$."

This - Albert and Loewer's "single mind view" [16] - gives only one sentient future Alice. To introduce a collection of present Alices who in future will experience each of the different possible experimental outcome, one could, instead, follow Albert and Loewer in postulating a continuum of Alice minds of which a proportion $\left|c_{0}\right|^{2}$ will see spin up. One could, in short, adopt the many-minds interpretation. I am not persuaded that there is a legitimate alternative formulation of Saunders' account.

\section{Appendix A: Further comments on counting descendants}

Wallace places great stress on the fact that there is no unique natural definition of a quasiclassical branch in an Everett universe, and so no way of counting the number of branches with any given feature. For example, a naive analysis of a quantum experiment with three possible outcomes might suggest that a single branch, pre-experiment, divides into three, post-experiment. But this, Wallace stresses, neglects the fact that quantum interactions take place very frequently in time and densely in space, outside our control. A careful attempt to quantify quasiclassical branches would show many branches splitting into many more during the lifetime of the experiment; however, there is no unique natural definition that would allow us to pin these numbers down. Hence, it is argued, there is no way of implementing the naive idea of using branch counts to define a rational strategy - an approach which would, if it worked, be a coherent alternative to the Born-rule-dependent strategy, and so refute the claim that the latter is the unique rational strategy.

There is indeed no known natural way of characterising and counting branches. It is worth reconsidering, however, whether there may nonetheless be a natural way for an agent about to observe an experiment to characterise and hence count his descendants, by considering their memory states after the experiment. 
Consider first a simple model of an observer, apparatus and quantum state which evolve unitarily during an experiment so that

$$
\left|O_{0}>\right| A_{0}>\left|Q_{0}>\rightarrow \sum_{j=1}^{3} a_{j}\right| O_{j}>\left|A_{j}>\right| Q_{j}>
$$

where the first state is at time 0 and the second at time $t$, the state $\left|A_{j}\right\rangle$ is the apparatus state registering outcome $j$ and $\mid O_{j}>$ is the observer state having observed the apparatus registering outcome $j$. The agent in state $\mid O_{0}>$ can reasonably say that he will have three successors $\mid O_{j}>$ at time $t$, corresponding to his three future distinct brain states.

Now consider a more detailed model in which we include an environment, and suppose that

$$
\left|O_{0}>\right| A_{0}>\left|Q_{0}>\right| E_{0}>\rightarrow \sum_{j=1}^{3} \sum_{i=1}^{n_{j}} a_{i j}\left|O_{j}>\right| A_{i j}>\left|Q_{j}>\right| E_{i j}>.
$$

Again, the sum over $j$ represents the three possible observer states. The sums over $i$ represent decompositions into orthogonal quasiclassical branches, and the number of terms $n_{j}$ in each sum depends on an arbitrary choice of definition of quasiclassical branch from among many possible definitions. Since this is supposed to be a model of the same experiment, we have that $\sum_{i}\left|a_{i j}\right|^{2}=\left|a_{j}\right|^{2}$ for $j=1,2,3$. The agent in state $\mid O_{0}>$ has no unique natural way of characterising the branches. However, he could consistently view each of the three components, containing the state $\mid O_{j}>, j=1,2,3$, as representing precisely one successor.67 After all, he is interested in his successors' welfare, and this is determined by their mind states; at this point in the analysis, at least, it is not affected by the state of the rest of the universe.

It might be objected that we still have not taken sufficiently into account the pervasiveness of environment-induced quantum interactions, which will presumably also be taking place within the agent's brain during the experiment. A more detailed model still would replace the sums on the right hand side of (A2) by sums including at least small components of a variety of different agent mind states, corresponding to different brain states that arise through zapping by stray cosmic rays and other quantum effects.

A related objection is that this way of counting successors leads to ambiguities when sequences of experiments are carried out. Suppose that the agent will carry out a second experiment, with two possible outcomes, if he observes outcome 1 in the first experiment, but not otherwise. After the first experiment but before the second, it appears that he has three successors, whose welfare he should value equally. After the second experiment, it appears that he has four successors, whose welfare he should again value equally. But since two of these descend from one of the original successors, and each "inherit" any resources he "bequeaths" to that successor, the two ways of counting lead to different allocation strategies - i.e. they disagree on which "bets" he should be willing to accept on the experiment.

To these objections, however, the agent could make several responses.

First, that his policy is not incoherent, but merely so far incompletely specified. In the case of the two experiments, he does care equally about the welfare of his three successors between the experiments, and he also cares equally about the welfare of his four successors after the experiments. To formulate a more precise policy, he would need to set out some way of trading off the welfares of different successors at different times: for example, by summing the time-integrated welfares over each distinct life-time segment. That this may become complicated doesn't imply that the aim is not rational. Indeed, we face very similar problems in worrying about the well-being of our genetic descendants. It is perfectly rational to value the welfare of all of your children equally, and also perfectly rational to value the welfare of all of your as yet unborn grandchildren equally, in both cases ceteris paribus. However, finding a rational asset allocation policy that respects both these preferences may require some further policy decisions, complicated calculations and predictions of uncertain future events.

Second, that he would indeed take into account the welfare of all his successors, including those whose mind states differ because of environmentally induced interactions, if he could. Here again, he can maintain that he has a rational policy in principle, albeit one that he cannot fully implement it in practice because of the impracticality of carrying out the relevant calculations. And again, he can say that the latter caveat does not detract from the rationality of his goal.

Third, that a consistent strategy for weighting his concern for the welfare of successors could be defined by considering the branching structure recorded in their own memory states. In the example above, successors who experience two experiments in succession remember that fact, and this distinguishes them from successors who experience only one experiment. It's logically consistent - and not obviously any more absurd than any Born-weight-dependent many-worlds strategy - to assign the former caring weight $1 / 6$ and the latter caring weight $1 / 3$.

To these responses, Everettians might in turn object that there is no natural way, even in principle, of characterising and counting all the possible mind states of successors of an agent exposed to real-world environmental interactions. 
But if the Everettian case eventually turns on this point, then the objection to rational strategies based on counting successors ultimately arises from an intrinsic vagueness in the quasifunctionalist theory of mind attached to the quantum formalism by Wallace et al., not, as claimed, from the vagueness in the notion of a quasiclassical branch. It seems a most uncomfortable defence of a purportedly fundamental theory to say that it is not well enough developed for us to be able to assess whether or not one of the key arguments advanced in its favour is valid.

\section{Appendix B: Further Comment on physical laws of rational compulsion}

What could it possibly mean to believe that the laws of physics per se rationally compel a particular behaviour for rational agents in a branching multiverse? The idea here, to be clear, is not merely the truism that the laws of physics imply significant facts about the world which rational agents might, or even must, sensibly take into account. It is that there are basic postulates, on an equal footing with other physical laws, that state by fiat that a particular type of behaviour is rationally compulsory for rational agents. I don't think I know what this can mean - the idea of such a law isn't consistent with my understanding of either physics or rationality - but the idea is definitely in play in some discussions of many-worlds theories in this volume. Papineau[22] proposes an axiom of this type, and Greaves-Myrvold [24] consider how to (purportedly) confirm theories including such axioms. My impression is that many Everettians share Greaves' view [23] that resorting to such a postulate would be, at least, an adequate fall-back should Wallace's [20] and other arguments not hold up.

Here's a point that seems not to have been considered in the Everettian literature. If we were to take seriously the idea that physical axioms can rationally compel rational beings to act in a certain way - by fiat, without further justification - then we must also take seriously the possibility that these rationally compelling axioms can take unfamiliar forms. For instance, in our branching universe, there's no reason to restrict to axioms that require rational preferences to be given by the ordering of values of expressions of the form $\sum_{i} p_{i} U_{i}$, where $U_{i}$ is the agent's utility for the outcome on branch $i$ and the $p_{i}$ are positive branch weights satisfying the Kolmogorov axioms. There's nothing logically inconsistent about postulating laws with negative or complex $p_{i}$, or preference orderings given by general joint functions $f\left(p_{i}, U_{i}\right)$, or indeed any other mathematical structure one cares to dream up. 68]

Such laws would generally violate Savage's axioms and perhaps other cherished intuitions about rational behaviour. But once one enters the strange game of postulating physical laws defining rational behaviour in multiverses, one needn't restrict one's postulates to intuitions developed in an attempt to provide a foundation for decision theory in a single chancy universe. Everettians who miss this point seem to me like hypothetical seventeenth century theorists who learn Hooke's law, come up with the idea that one can postulate abstract physical force laws that define forces between objects unmediated by springs, but then still maintain that these laws necessarily have to set force proportional to separation. Their boldness is inconsistently selective: an abstract law need not be constrained by the details of the concrete model that inspired it.

Of course, Everettians who think it makes sense to postulate laws of rational compulsion still have the option of basing their postulates on one-world probability theory, and specifically on optimising Born-rule-weighted average utility. But one needs to be clear that, prima facie, this is an arbitrary choice from a very large range of possibilities. Along with everything else in this peculiar game, that choice seems to lack justification. Moreover, as the above analysis of Greaves-Myrvold's account of confirmation applied to the weightless universe shows, allowing arbitrary choices of laws of rational compulsion means not only that many mutually inconsistent choices can be postulated in the same multiverse but also that each of them can be, by their own lights, confirmed.

\section{Appendix C: A possible empirical distinction between many-worlds and one-world quantum theory}

Finally, suppose, notwithstanding all the arguments above, that we arrive at an Everettian theory that, while perhaps ad hoc and unattractive, is coherent - for example, some version of the many-minds interpretation 16 . It is generally believed that, without very advanced technology which allows the re-interference of macroscopically distinct branches, such a theory will necessarily be empirically indistinguishable from Copenhagen quantum theory.

The following argument against this conclusion relies on anthropic reasoning and also on the hypothesis that species may evolve a consistent preference for or against higher population expectation over higher survival probability. Anthropic reasoning is notoriously tricky to justify, and we may anyway not necessarily have evolved demonstrable consistent preferences one way or the other, so the argument may not necessarily have practical application. Nonetheless, it does show in principle that evolutionary evidence could make many-worlds theories more or less plausible.

Consider a simple model of two species $A$ and $B$, both of which begin with population $P$ and are offered, each year, the option of doing something that depends on a quantum event and carries a 0.5 probability of extinction and a 0.5 probability of trebling the species population. Suppose that, if they reject the option, their population remains 
constant, as it does in between these decisions. Species $A$ is risk-averse, and so always declines the option. Species $B$ is risk-tolerant, and instinctively driven to maximise expected population, and so always accepts.

Now let $N$ be a large integer. After $N$ years, if one-world quantum theory is correct, species $A$ will have population $P$, and species B will have either population 0 (with probability $\left(1-\left(\frac{1}{2}\right)^{N}\right)$ ) or population $3^{N}$ (with probability $\left(\frac{1}{2}\right)^{N}$ ). In other words, species $B$ will almost surely be extinct. If these are the only two species, and you are alive in the $N$-th year, almost certainly you belong to species $A$.

If many-worlds quantum theory is correct, species $A$ still has population $P$ in all branches. Species $B$ has population 0 in branches of total Born weight $\left(1-\left(\frac{1}{2}\right)^{N}\right)$, and population $3^{N}$ in branches of total Born weight $\left(\frac{1}{2}\right)^{N}$. Now, if anthropic reasoning is justifiable here, and you are alive in the $N$-th year, almost certainly you belong to species $B$. (There are $\left(\frac{3}{2}\right)^{N}$ times as many minds belonging to species $B$ as to $A$ after $N$ years.)

In other words, there is a sense in which long-run evolutionary success is defined by different measures in one-world and many-worlds quantum theory. If anthropic reasoning were justifiable, then one could in principle infer whether one-world or many-worlds quantum theory is likelier correct by seeing whether one belongs to a Born-weighted expected population maximising species or to a risk-averse species that seeks to maximise its Born-weighted survival probability. Readers may thus wish to consider whether their species has evolved a coherent strategy of either type. 69.

\section{Acknowledgments}

I am very grateful to Jonathan Barrett for many thoughtful and constructive comments on and criticisms of a preliminary version of the manuscript, as well as other very valuable conversations, and also to Hilary Greaves and David Wallace for patiently tolerating and helpfully engaging with my critical probing. Thanks too to David Albert, Harvey Brown, Jeremy Butterfield, Chris Fuchs, Lucien Hardy, Graeme Mitchison, Wayne Myrvold, David Papineau, Huw Price, Simon Saunders, Tony Short, John Sipe, Rob Spekkens and Tony Sudbery for some valuable conversations. This research was partially supported by a grant from The Foundational Questions Institute (fqxi.org) and by Perimeter Institute for Theoretical Physics. Research at Perimeter Institute is supported by the Government of Canada through Industry Canada and by the Province of Ontario through the Ministry of Research and Innovation.

[1] H. Everett, Relative State Formulation of Quantum Mechanics, Reviews of Modern Physics 29 (1957) pp. 454-462; reprinted in Ref. 9], pp. 141-149.

[2] H. Everett, The Theory of the Universal Wave Function, in Ref. [9], pp. 3-140.

[3] A. Kent, A Solution to the Quantum Reality Problem, in preparation.

[4] J.L. Borges, The Garden of Forking Paths, Ellery Queen's Mystery Magazine (August 1948); reprinted in J.L. Borges, Collected Fictions: Ficciones (Viking, New York, 1999).

[5] P.K. Feyerabend, How To Defend Society Against Science, Radical Philosophy 11 (1975) 277-283.

[6] J. Hartle, Quantum Mechanics of Individual Systems, American Journal of Physics 36, 704-712 (1968).

[7] B. DeWitt, Quantum Mechanics and Reality and The Many-Universes Interpretation of Quantum Mechanics, in Ref. [9], pp. 155-166 and 167-218.

[8] N. Graham, The Measurement of Relative Frequency, in Ref. 9], pp. 229-253.

[9] B. DeWitt and N. Graham (eds.), The Many-Worlds Interpretation of Quantum Mechanics, (Princeton University Press, Princeton, 1973).

[10] R. Geroch, The Everett Interpretation, Noûs 18 (1984), 617-633.

[11] D. Deutsch, Quantum Theory as a Universal Physical Theory, Int. J. Theor. Phys. 24 1-41 (1985).

[12] D. Deutsch, Quantum Theory of Probability and Decisions, Proc. Roy. Soc. Lond. A455 (1999) 3129-3137.

[13] S. Saunders, Chance in the Everett Interpretation, this volume.

[14] J. Barbour, The End of Time, Oxford University Press (2001); and refs. therein.

[15] J.S. Bell, The Measurement Theory of Everett and De Broglie's Pilot Wave and Quantum Mechanics for Cosmologists, in Speakable and Unspeakable in Quantum Mechanics, Cambridge University Press (1987).

[16] D. Albert and B. Loewer, Interpreting the many worlds interpretation, Synthese 77 (1988) 195-213.

[17] S. Coleman, Quantum Mechanics in Your Face, lecture video archived at http://media.physics.harvard.edu/video/index.php?id=SidneyColeman_QMIYF.flv (1994).

[18] M. Lockwood, 'Many Minds' Interpretations of Quantum Mechanics, Brit. J. Philos. Sci 47 (1996), 159-188.

[19] D. Wallace, Decoherence and Ontology, this volume.

[20] D. Wallace, How to prove the Born rule, this volume.

[21] L. Vaidman, Many-worlds Interpretation of Quantum Mechanics, Stanford Encyclopaedia of Philosophy online article at plato.stanford.edu (2002); Time Symmetry and the Many-Worlds Interpretation, this volume.

[22] D. Papineau, A Fair Deal for Everettians, this volume; and references therein. 
[23] H. Greaves, Understanding Deutsch's probability in a deterministic multiverse, Studies in History and Philosophy of Modern Physics 35 (2004) pp.423-456.

[24] H. Greaves and W. Myrvold, Everett and Evidence, this volume.

[25] M. Gell-Mann and J. Hartle, Classical Equations for Quantum Systems, Phys. Rev. D47 (1993) 3345-3382; J. Hartle, this volume.

[26] W. Zurek, From Relative States to Quantum Jumps, Born's Rule, and Objective Reality, this volume; and references therein.

[27] M. Tegmark, Many Worlds in Context, this volume.

[28] F. Dowker and A. Kent, Properties of Consistent Histories, Phys. Rev. Lett. 75 (1995) 3038-3041.

[29] F. Dowker and A. Kent, On the Consistent Histories Approach to Quantum Mechanics, J. Stat. Phys. 82 (1996) $1575-1646$.

[30] A. Kent, Consistent Sets Yield Contrary Inferences in Quantum Theory, Phys. Rev. Lett. 78 (1997) 2874-2877; Reply to Griffiths and Hartle, Phys. Rev. Lett. 81 (1998) 1982.

[31] A. Kent, Quantum Histories, Phys. Scripta T76 (1998) 78-84.

[32] A. Kent, Quantum Histories and their Implications, in Relativistic Quantum Measurement and Decoherence, F. Petruccione (ed.), Lecture Notes in Physics 559 93-115 (Springer-Verlag, Berlin, 2000).

[33] A. Kent, Quasiclassical Dynamics in a Closed Quantum System, Phys. Rev. A 54 (1996) 4670-4675.

[34] D. Albert, Probability in the Everett Picture, this volume.

[35] H. Price, Decisions, Decisions, Decisions: Can Savage Salvage Everettian Probability?, this volume.

[36] J.S. Rawls, A Theory of Justice, Harvard University Press, Cambridge, Massachusetts (1971).

[37] H. Barnum et al., Quantum Probability from Decision Theory?, Proc. Roy. Soc. Lond. A456 (2000) 1175-1182.

[38] A. Kent, A critical look at risk assessments for global catastrophes, Risk Analysis 24 (2004) 157-168.

[39] See the discussion of "The variety rule" in Ref. [20].

[40] In section 5 of Ref. [20].

[41] L. Savage, The foundations of statistics, (Dover, New York, 1972).

[42] On page 5 of Ref. [41].

[43] K.J. Arrow, A Difficulty in the Concept of Social Welfare, Journal of Political Economy 58 (1950) 328-346.

[44] Savage makes a related point in criticising his own approach: see Chap. 4 of Ref. [41].

[45] A. Kent, Against Many-Worlds Interpretations, Int. J. Mod. Phys. A5 (1990) 1745.

[46] Y. Aharonov, B. Bergman, and J. Leibowitz, Time symmetry in the quantum process of measurement, Phys. Rev. B 134, (1964) 1410-1416.

[47] M. Gell-Mann and J. Hartle, Time Symmetry and Asymmetry in Quantum Mechanics and Quantum Cosmology, in Physical Origins of Time Asymmetry, J. Halliwell, J. Perez-Mercader and W. Zurek (eds.), Cambridge University Press, Cambridge, (1994).

[48] S. Saunders, Time, Quantum Mechanics and Probability, Synthese 114, (1998) 405-444.

[49] S. Saunders and D. Wallace, Branching and Uncertainty, Brit. J. Phil. Sci. 59, (2008) 293-305.

[50] D. Wallace, Epistemology Quantized: circumstances in which we should come to believe in the Everett interpretation, Brit. J. Phil. Sci. 57 (2006) 655-689.

[51] D. Papineau, Rational Decisions and The Many Minds Interpretation of Quantum Mechanics, The Monist 80 (1997) 97-117; reprinted in D. Papineau, The Roots of Reason: Philosophical Essays on Rationality, Evolution and Probability, Oxford University Press (2003), pp. 212-239.

[52] H. Greaves, Understanding Deutsch's probability in a deterministic multiverse, Stud. Hist. Phil. Mod. Phys. 35 (2004) 423-456.

[53] I thank Jonathan Barrett for this point and this example.

[54] There are more general possibilities.

[55] See Appendix B] for further discussion.

[56] That all ambiguities in total weights of quasiclassical outcomes are necessarily very small seems plausible and is what Wallace expects. Given the level of conceptual imprecision in discussing the emergence of quasiclassical structures from unitary quantum theory, though, it is hard to be certain even of this.

[57] And to run such an argument, Wallaceans would, inter alia, need to find suitable precise definitions of "closer" and "better off".

[58] Given the fuzzy ontology, we should more accurately say "to any possible assignment of the imprecisely defined value of the true mean utility". For the sake of readability, we take this qualification as read in what follows.

[59] Without these assumptions, the mean utilitarian's strategy isn't defined, in which case Wallace's argument has failed.

[60] Of course, in both these last two cases, it could still be a problem if the numbers so conspire.

[61] I thank Hans Westman and Ward Struyve for suggesting this example.

[62] Or perhaps, considering the uneasiness of crown-wearing heads, vice versa.

[63] Wallace 20] notes and discusses the special case of the Price-Rawlsian.

[64] Incidentally, this issue has also divided Everettians: Papineau [51] and, at one point, Greaves [52], have also argued that subjective uncertainty is not to be had in Everettian quantum theory.

[65] My remarks here follow Albert's lucid discussion [34].

[66] The same is true in more realistic models.

[67] He is not rationally compelled to accept this view. The point is that in this model successor counting is mathematically well-defined and hence it's possible to use it to define a rational strategy.

[68] Indeed, one could imagine an exotic story about inverted qualia and hence reversing of utilities on some branches, which justifies giving them negative weights. And perhaps, some might argue, the complex quantum amplitudes defining the path 
integral should be interpreted as directly defining rational constraints on an agent's preferences for the entire set of paths defining a hypothetical future unitary evolution.

[69] Unfortunately, I suspect mine has not. 